DOE/ID-22245

Prepared in cooperation with the U.S. Department of Energy

\title{
Correlation between Basalt Flows and Radiochemical and Chemical Constituents in Selected Wells in the Southwestern Part of the Idaho National Laboratory, Idaho
}

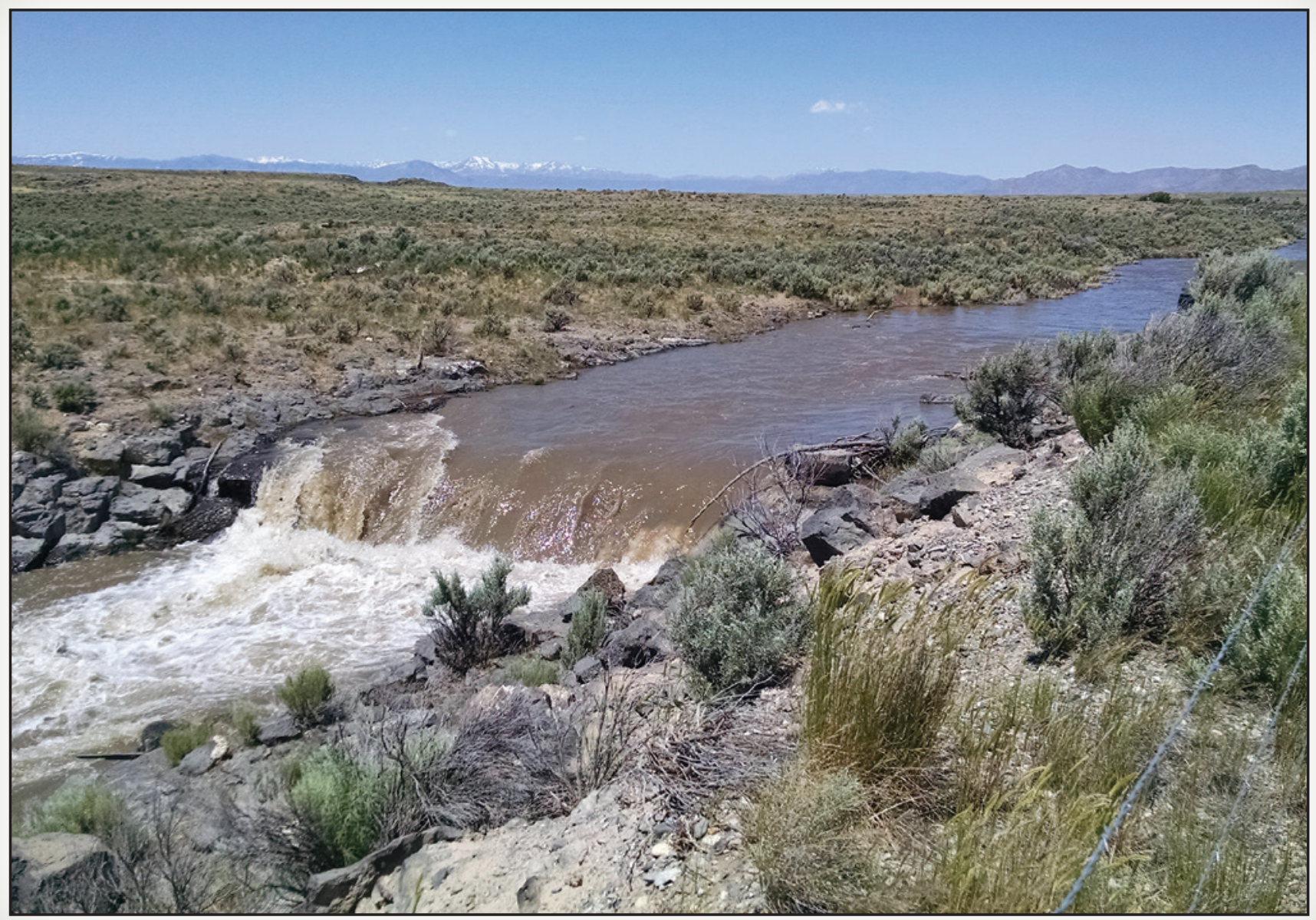

Scientific Investigations Report 2017-5148 
Cover: Photograph showing view of the Big Lost River flowing into the Idaho National Laboratory spreading areas, Idaho. Photograph by Roy Bartholomay, U.S. Geological Survey, June 22, 2017. 


\section{Correlation between Basalt Flows and Radiochemical and Chemical Constituents in Selected Wells in the Southwestern Part of the Idaho National Laboratory, Idaho}

By Roy C. Bartholomay, Mary K.V. Hodges, and Duane E. Champion

DOE/ID-22245

Prepared in cooperation with the U.S. Department of Energy

Scientific Investigations Report 2017-5148 


\title{
U.S. Department of the Interior \\ RYAN K. ZINKE, Secretary
}

\section{U.S. Geological Survey William H. Werkheiser, Deputy Director exercising the authority of the Director}

\author{
U.S. Geological Survey, Reston, Virginia: 2017
}

For more information on the USGS - the Federal source for science about the Earth, its natural and living resources, natural hazards, and the environment-visit https://www.usgs.gov or call 1-888-ASK-USGS.

For an overview of USGS information products, including maps, imagery, and publications, visit https://store.usgs.gov.

Any use of trade, firm, or product names is for descriptive purposes only and does not imply endorsement by the U.S. Government.

Although this information product, for the most part, is in the public domain, it also may contain copyrighted materials as noted in the text. Permission to reproduce copyrighted items must be secured from the copyright owner.

Suggested citation:

Bartholomay, R.C., Hodges, M.K.V., and Champion, D.E., 2017, Correlation between basalt flows and radiochemical and chemical constituents in selected wells in the southwestern part of the Idaho National Laboratory, Idaho: U.S. Geological Survey Scientific Investigations Report 2017-5148 (DOE/ID-22245), 39 p., https://doi.org/10.3133/ sir20175148.

ISSN 2328-0328 (online) 


\section{Contents}

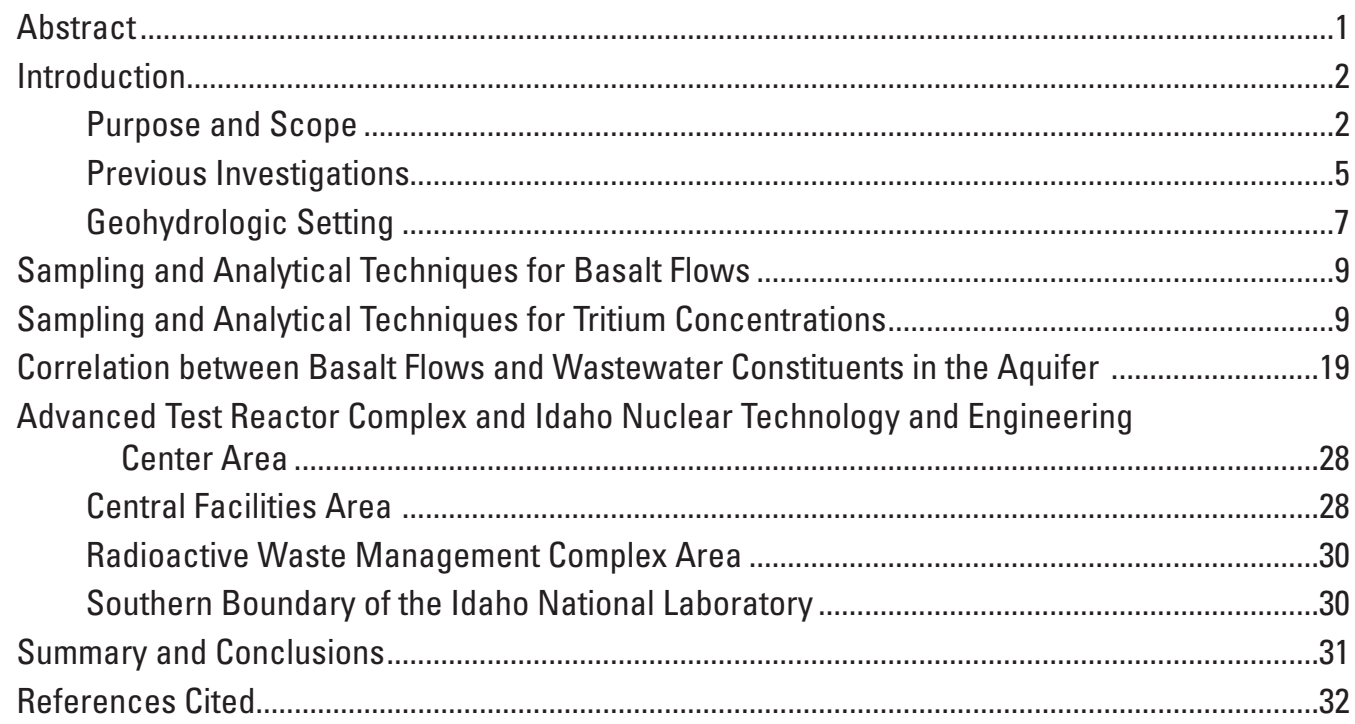

Appendix A. Paleomagnetic Inclination Values and Basalt Flow from Coreholes in the

Eastern Snake River Plain Aquifer at the Idaho National Laboratory

\section{Figures}

1. Map showing location of the study area, selected facilities, volcanic vents, coreholes, and the Arco-Big Southern Butte and Axial volcanic zones, southwestern part of the Idaho National Laboratory, Idaho

2. Map showing location of wells sampled for radiochemical and chemical constituents, Idaho National Laboratory, Idaho …………...............................................

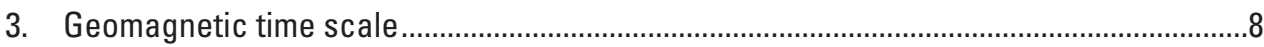

4. Map showing tritium concentrations in water sampled from wells in the southwestern part of the Idaho National Laboratory, Idaho

5. Maps showing occurrence of basalt flows Big Lost, CFA Buried Vent, AEC Butte, Late Basal Brunhes, Middle Basal Brunhes, Early Basal Brunhes, South Late Matuyama, and Matuyama in wells in the southwestern part of the Idaho National Laboratory, Idaho

6. Map showing hydraulic conductivity measured in selected wells in the southwestern part of the Idaho National Laboratory, Idaho

\section{Tables}

1. Summary of selected previous investigations about geology, water quality, paleomagnetism, and stratigraphy of the eastern Snake River Plain and Idaho National Laboratory, Idaho.

2. Data from selected coreholes and groundwater sampling sites at the Idaho National Laboratory, Idaho

3. Hydraulic conductivity, tritium, and chloride data for selected groundwater sampling sites at the Idaho National Laboratory, Idaho

4. Stratigraphic sequence and percentage of flows influenced by wastewater at the Idaho National Laboratory, Idaho 


\section{Conversion Factors}

U.S. customary units to International System of Units

\begin{tabular}{|c|c|c|}
\hline Multiply & By & To obtain \\
\hline \multicolumn{3}{|c|}{ Length } \\
\hline inch (in.) & 2.54 & centimeter $(\mathrm{cm})$ \\
\hline foot $(\mathrm{ft})$ & 0.3048 & $\operatorname{meter}(\mathrm{m})$ \\
\hline mile (mi) & 1.609 & kilometer (km) \\
\hline \multicolumn{3}{|c|}{ Area } \\
\hline acre & 4,047 & square meter $\left(\mathrm{m}^{2}\right)$ \\
\hline square foot $\left(\mathrm{ft}^{2}\right)$ & 0.09290 & square meter $\left(\mathrm{m}^{2}\right)$ \\
\hline square mile $\left(\mathrm{mi}^{2}\right)$ & 2.590 & square kilometer $\left(\mathrm{km}^{2}\right)$ \\
\hline \multicolumn{3}{|c|}{ Volume } \\
\hline cubic mile $\left(\mathrm{mi}^{3}\right)$ & 4.168 & cubic kilometer $\left(\mathrm{km}^{3}\right)$ \\
\hline \multicolumn{3}{|c|}{ Hydraulic conductivity } \\
\hline foot per day (ft/d) & 0.3048 & meter per day $(\mathrm{m} / \mathrm{d})$ \\
\hline \multicolumn{3}{|c|}{ Hydraulic gradient } \\
\hline foot per mile (ft/mi) & 0.1894 & meter per kilometer $(\mathrm{m} / \mathrm{km})$ \\
\hline \multicolumn{3}{|c|}{ Transmissivity } \\
\hline foot squared per day $\left(\mathrm{ft}^{2} / \mathrm{d}\right)$ & 0.09290 & meter squared per day $\left(\mathrm{m}^{2} / \mathrm{d}\right)$ \\
\hline
\end{tabular}

\section{Datums}

Vertical coordinate information is referenced to the North American Vertical Datum of 1988 (NAVD 88).

Horizontal coordinate information is referenced to the North American Datum of 1927 (NAD 27). Altitude, as used in this report, refers to distance above the vertical datum.

\section{Supplemental Information}

Transmissivity: The standard unit for transmissivity is cubic foot per day per square foot times foot of aquifer thickness $\left(\left[\mathrm{ft}^{3} / \mathrm{d}\right] / \mathrm{ft}^{2}\right) \mathrm{ft}$. In this report, the mathematically reduced form, foot squared per day $\left(\mathrm{ft}^{2} / \mathrm{d}\right)$, is used for convenience.

Concentrations of chemical constituents in water are given in either milligrams per liter (mg/L) or micrograms per liter ( $\mu \mathrm{g} / \mathrm{L})$.

Activities for radioactive constituents in water are given in picocuries per liter ( $\mathrm{pCi} / \mathrm{L})$. 


\title{
Abbreviations
}

\author{
AEC Atomic Energy Commission \\ ATR Advanced Test Reactor \\ AVZ Axial Volcanic Zone \\ CFA Central Facilities Area \\ DOE Department of Energy \\ EBB Early Basal Brunhes \\ ESRP Eastern Snake River Plain \\ ICPP Idaho Chemical Processing Plant \\ INL Idaho National Laboratory \\ INTEC Idaho Nuclear Technology and Engineering Center \\ LBB Late Basal Brunhes \\ M Matuyama \\ $\mathrm{Ma} \quad$ million years \\ MBB Middle Basal Brunhes \\ NPR New Production Reactor \\ NRF Naval Reactors Facility \\ RWMC Radioactive Waste Management Complex \\ SDA Subsurface Disposal Area \\ SLM South Late Matuyama \\ SRP Snake River Plain \\ TAN Test Area North \\ USGS U.S. Geological Survey
}





\title{
Correlation between Basalt Flows with Radiochemical and Chemical Constituents in Selected Wells in the Southwestern Part of the Idaho National Laboratory, Idaho
}

\author{
By Roy C. Bartholomay, Mary K.V. Hodges, and Duane E. Champion
}

\begin{abstract}
Wastewater discharged to wells and ponds and wastes buried in shallow pits and trenches at facilities at the Idaho National Laboratory (INL) have contributed contaminants to the eastern Snake River Plain (ESRP) aquifer in the southwestern part of the INL. This report describes the correlation between subsurface stratigraphy in the southwestern part of the INL with information on the presence or absence of wastewater constituents to better understand how flow pathways in the aquifer control the movement of wastewater discharged at INL facilities. Paleomagnetic inclination was used to identify subsurface basalt flows based on similar inclination measurements, polarity, and stratigraphic position. Tritium concentrations, along with other chemical information for wells where tritium concentrations were lacking, were used as an indicator of which wells were influenced by wastewater disposal.
\end{abstract}

The basalt lava flows in the upper 150 feet of the ESRP aquifer where wastewater was discharged at the Idaho Nuclear Technology and Engineering Center (INTEC) consisted of the Central Facilities Area (CFA) Buried Vent flow and the AEC Butte flow. At the Advanced Test Reactor (ATR) Complex, where wastewater would presumably pond on the surface of the water table, the CFA Buried Vent flow probably occurs as the primary stratigraphic unit present; however, AEC Butte flow also could be present at some of the locations. At the Radioactive Waste Management Complex (RWMC), where contamination from buried wastes would presumably move down through the unsaturated zone and pond on the surface of the water table, the CFA Buried Vent; Late Basal Brunhes; or Early Basal Brunhes basalt flows are the flow unit at or near the water table in different cores.

In the wells closer to where wastewater disposal occurred at INTEC and the ATR-Complex, almost all the wells show wastewater influence in the upper part of the ESRP aquifer and wastewater is present in both the CFA Buried Vent flow and AEC Butte flow. The CFA Buried Vent flow and AEC Butte flow are also present in wells at and north of CFA and are all influenced by wastewater contamination. All wells with the AEC Butte flow present have wastewater influence and 83 percent of the wells with the more prevalent CFA Buried Vent flow have wastewater influence. South and southeast of CFA, most wells are not influenced by wastewater disposal and are completed in the Big Lost Flow and the CFA Buried Vent flow. Wells southwest of CFA are influenced by wastewater disposal and are completed in the Big Lost flow and CFA Buried Vent flow at the top of the aquifer. Basalt stratigraphy indicates that the CFA Buried Vent flow is the predominant flow in the upper part of the ESRP aquifer at and near the RWMC as it is present in all the wells in this area. The Late Basal Brunhes flow, Middle Basal Brunhes flow, Early Basal Brunhes flow, South Late Matuyama flow, and Matuyama flow are also present in various wells influenced by waste disposal.

Some wells south of RWMC do not show wastewater influence, and the lack of wastewater influence could be due to low hydraulic conductivities. Several wells south and southeast of CFA also do not show wastewater influence. Low hydraulic conductivities or ESRP subsidence are possible causes for lack of wastewater south of CFA.

Multilevel monitoring wells completed much deeper in the aquifer show influence of wastewater in numerous basalt flows. Well Middle 2051 (northwest of RWMC) does not show wastewater influence in its upper three basalt flows (CFA Buried Vent, Late Basal Brunhes, and Middle Basal Brunhes); however, wastewater is present in two deeper flows (the Matuyama and Jaramillo flows). Well USGS 131A (southwest of CFA) and USGS132 (south of RWMC) both show wastewater influence in all the basalt flows sampled in the upper 600 feet of the aquifer. Wells USGS 137A, 105, 108, and 103 completed along the southern boundary of the INL all show wastewater influence in several basalt flows including the G flow, Middle and Early Basal Brunhes flows, the South Late Matuyama flow and the Matuyama flow; however, the strongest wastewater influence appears to be in the South Late Matuyama flow. The concentrations of wastewater constituents in deeper parts of these wells support the concept of groundwater flow deepening in the southwestern part of the INL. 


\section{Introduction}

The Idaho National Laboratory (INL), operated by the U.S. Department of Energy (DOE), encompasses about $890 \mathrm{mi}^{2}$ of the eastern Snake River Plain (ESRP) in southeastern Idaho (fig. 1). The INL was established in 1949 to develop atomic energy, nuclear safety, defense programs, environmental research, and advanced energy concepts. Wastewater disposal sites at the Test Area North (TAN), the Naval Reactors Facility (NRF), the Advanced Test Reactor Complex (ATR-Complex), and the Idaho Nuclear Technology and Engineering Center (INTEC) (fig. 1) have contributed radioactive- and chemical-waste contaminants to the ESRP aquifer. These sites incorporated various wastewater disposal methods, including lined evaporation ponds, unlined percolation (infiltration) ponds and ditches, drain fields, and injection wells. Waste materials buried in shallow pits and trenches within the Subsurface Disposal Area (SDA) at the Radioactive Waste Management Complex (RWMC) also have contributed contaminants to groundwater. Concern about subsurface movement of contaminants from these wastes increased the number and kinds of studies of subsurface geology and hydrology to provide information for conceptual and numerical models of groundwater flow and contaminant transport (Anderson and Lewis, 1989; Anderson, 1991; Anderson and Bartholomay, 1995; Anderson and Bowers, 1995; Anderson and Liszewski, 1997; Anderson, Ackerman, and others, 1996; Anderson, Liszewski, and Ackerman, 1996; Ackerman and others, 2006, 2010).

U.S. Geological Survey (USGS) scientists have been studying the geology, petrography, paleomagnetism, age of basalt flows, and hydrology of the ESRP for more than 100 years beginning with Russell (1902). Studies of the geologic framework of the ESRP at and near the INL intensified in 1949 when feasibility studies for siting of the INL began. Studies included evaluation of hydraulic properties of the aquifer, seismic and volcanic hazards, facility design and construction, and the evolution of basaltic volcanism on the ESRP.

Accumulations of basalt flows, eruptive fissures and vents, and fluvial and eolian sediments differ greatly in hydraulic conductivity, and the three-dimensional distribution of these materials control groundwater movement in the ESRP aquifer (Welhan and others, 2002). Basalt flows comprise more than 85 percent of the volume of the subsurface of the ESRP (Kuntz and others, 1992). Sedimentary interbeds comprise the rest. Paleomagnetic inclination and polarity studies on samples from subsurface drill cores and surface samples provide valuable data that can help constrain the age and extent of basalt flow groups. Data from paleomagnetic studies at and near the INL (Champion and Greeley, 1978; Champion and others, 1981; Champion and others, 1988) also have been used to document paleomagnetic secular variation
(Hagstrum and Champion, 2002) during late Pliocene to Holocene time. The Big Lost Reversed Polarity Cryptochron (formerly referred to as the Big Lost Reversed Polarity Subchron (Champion and others, 1988) of the Brunhes Normal Polarity Chron was first identified in subsurface ESRP basalts at the INL (Champion and others, 1981, 1988). This study was done by the U.S. Geological Survey (USGS) in cooperation with the DOE Idaho Operations Office.

\section{Purpose and Scope}

Knowledge about the subsurface geologic framework of the ESRP aquifer is being used to aid in refining conceptual and numerical models of groundwater flow and contaminant transport at and near the INL. Better definition of wastewater constituent movement in the aquifer in relation to the geologic framework also will aid in creating a solute transport model. This report describes the correlation between subsurface stratigraphy in the southwestern part of the INL and presents information about the presence or absence of wastewater constituents to help develop a better understanding of how wastewater discharged at INL facilities is controlled by flow pathways in the aquifer. Paleomagnetic inclination data were used to identify subsurface basalt flows based on similar inclination measurements, polarity, and stratigraphic position. The subcore plugs collected from drill cores and used in this study yield only paleomagnetic-inclination data because the original declination of the drill cores is not preserved during drilling. Other data, such as lithology, petrology, geophysical logs, and geochemistry, can be used in conjunction with paleomagnetic inclination, stratigraphic, and age data to confirm or reject correlations. The presence or absence of wastewater constituents was determined using tritium concentrations or other constituents such as chloride concentrations or organic compounds. Tritium concentrations were used because they are sampled in every well at the INL and have established background concentrations. Wells with tritium concentrations less than 75 picocuries per liter ( $\mathrm{pCi} / \mathrm{L}$ ) and (or) less than detection levels were not considered to be influenced by wastewater disposal unless organic compounds were present or chloride was present above background concentrations.

Paleomagnetic data were used to correlate surface and subsurface stratigraphy, determine relative ages, and, in conjunction with previous studies, determine the absolute age of certain basalt flows. Samples were collected from coreholes from depths of a few feet to 1,653 feet (ft). Drill core samples were selected from individual lava flow units based on identification of flow tops and bottoms in the coreholes. Well information was used to determine production zones that are correlated with the basaltic flow units. The wells considered for this study are in the southwestern part of the INL near and downgradient from the ATR Complex and INTEC (fig. 2). 


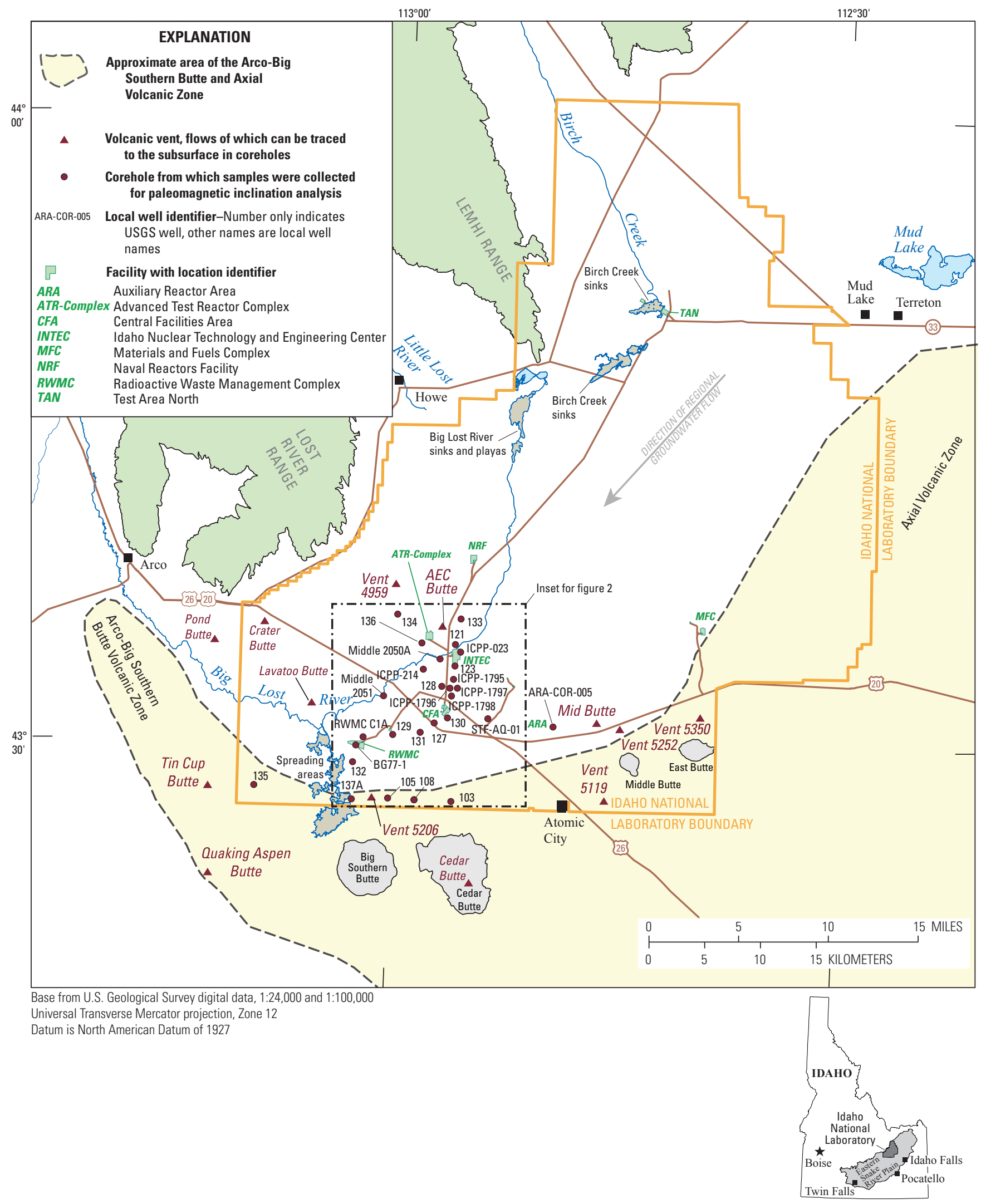

Figure 1. Location of the study area, selected facilities, volcanic vents, coreholes, and the Arco-Big Southern Butte and Axial volcanic zones, southwestern part of the Idaho National Laboratory, Idaho. 

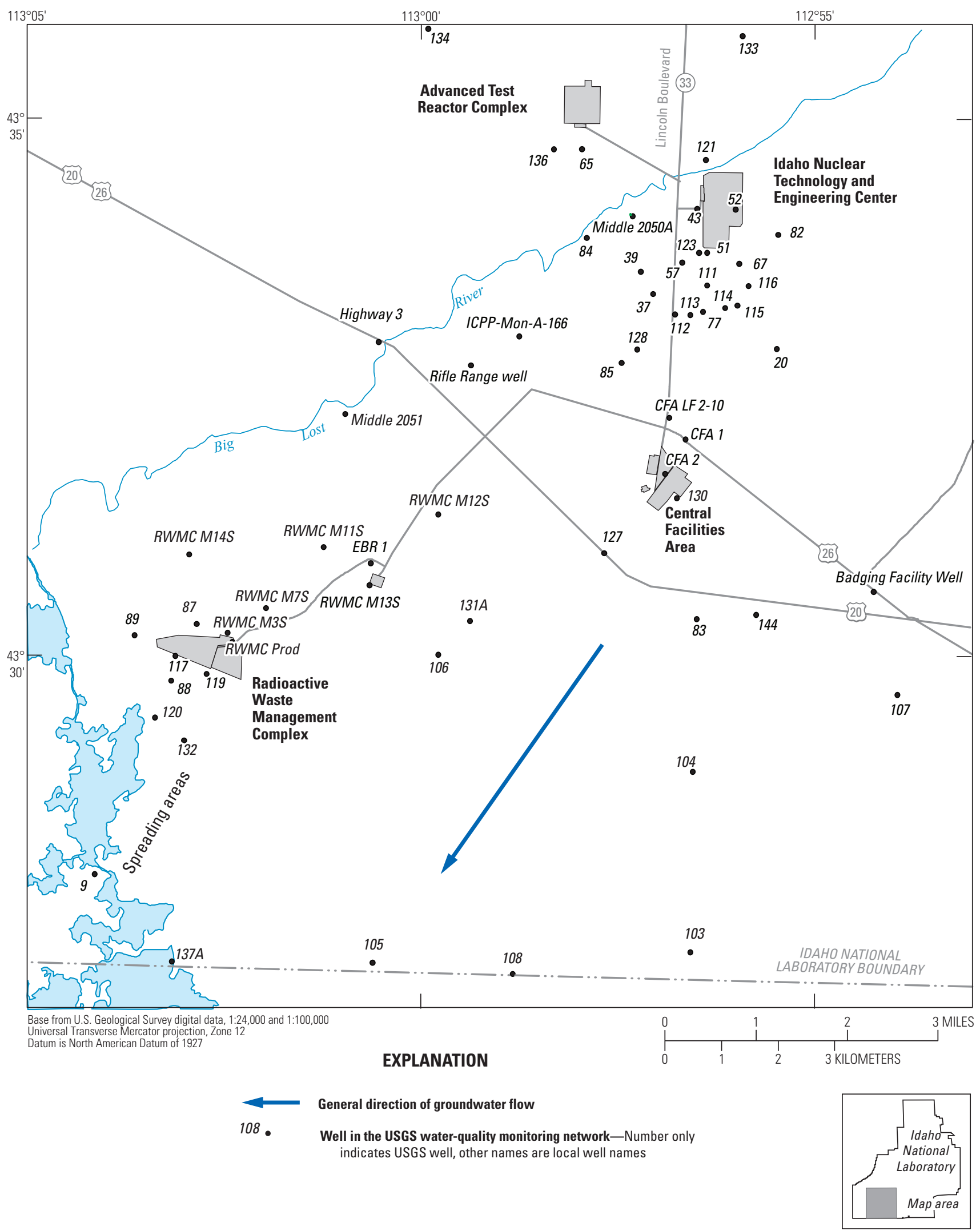

Figure 2. Location of wells sampled for radiochemical and chemical constituents, Idaho National Laboratory, Idaho. 


\section{Previous Investigations}

Numerous investigations of water quality in the ESRP aquifer and geologic, paleomagnetic, and stratigraphic investigations on surface and subsurface basalts at and near the INL and the ESRP have been completed. Selected previous investigations and the areas of investigation are listed in table 1. Paleomagnetic data records the magnetic field at the time of eruption and is not unique. Other data such as lithology, petrology, geophysical logs, and geochemistry must be used in conjunction with paleomagnetic and age data to confirm or reject correlations.

The stratigraphic framework for the conceptual model for groundwater flow, published in 2006 (Ackerman and others, 2006) was based on a limited number of cores, and natural gamma geophysical logs from uncored wells (Anderson and Lewis, 1989; Anderson, 1991; Anderson and Bartholomay, 1995; Anderson and Bowers, 1995; Anderson, Ackerman, and others, 1996; Anderson, Liszewski, and Ackerman, 1996; Anderson and Liszewski, 1997; Anderson and others, 1999). The subsurface stratigraphy proposed by Anderson and his co-workers, has been largely confirmed by recent paleomagnetic stratigraphy studies (Champion and others, 2011, 2013; Hodges and Champion, 2016). This report presents a more detailed comparison of the stratigraphy through the southwestern part of the site with the location of groundwater contamination that was evaluated in several recent studies (Bartholomay and others, 2015, 2017; Davis and others, 2015; Bartholomay and Hall, 2016).

Table 1. Summary of selected previous investigations about geology, water quality, paleomagnetism, and stratigraphy of the eastern Snake River Plain and Idaho National Laboratory, Idaho.

[Abbreviations: ATR, Advanced Test Reactor; CFA, Central Facilities Area; ESRP, eastern Snake River Plain; INL, Idaho National Laboratory; INTEC, Idaho Nuclear Technology and Engineering Center (also known as ICPP [Idaho Chemical Processing Plant]); NPR, New Production Reactor; NRF, Naval Reactors Facility; RWMC, Radioactive Waste Management Complex; SRP, Snake River Plain; TAN, Test Area North; USGS; U.S. Geological Survey]

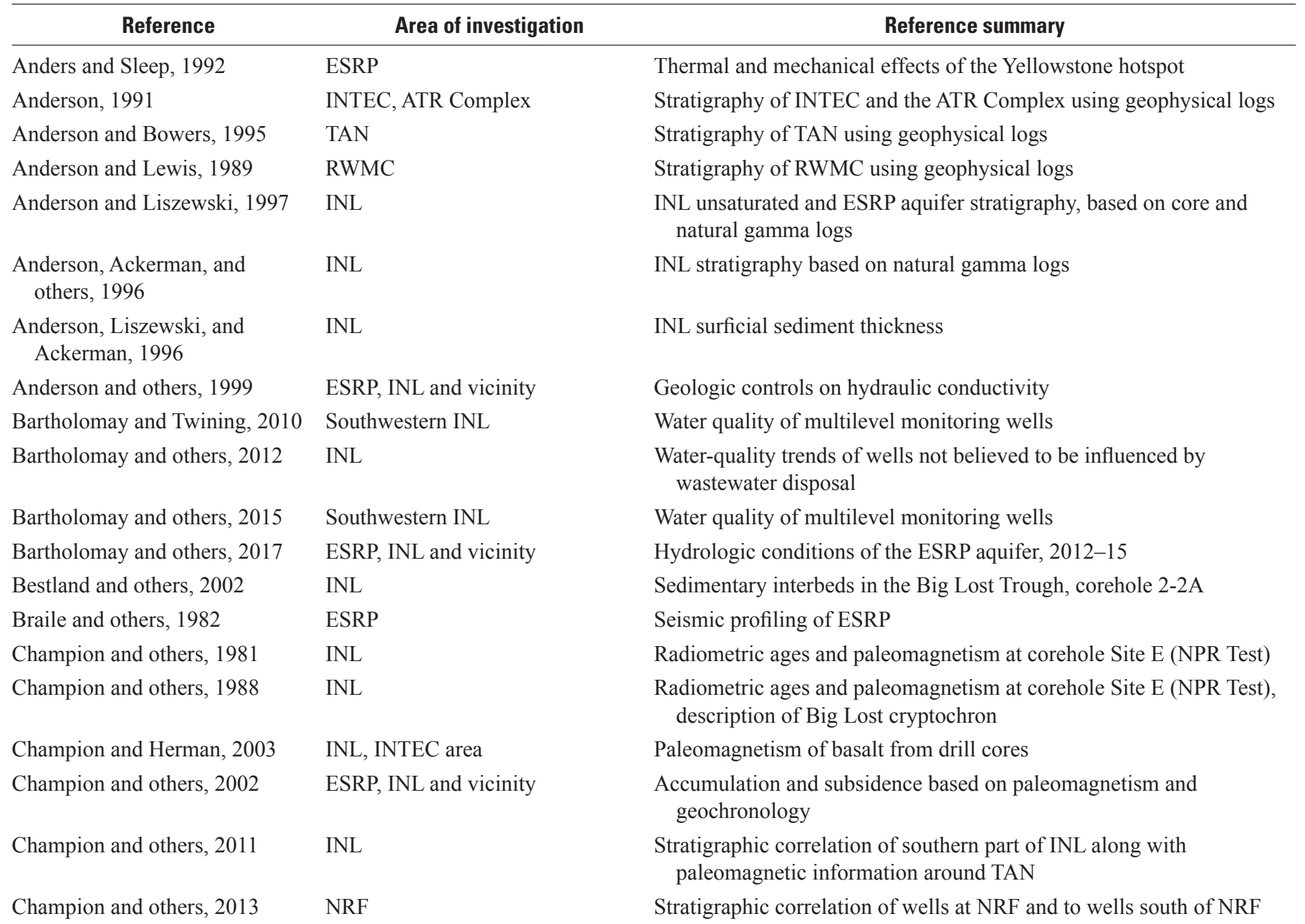


Table 1. Summary of selected previous investigations on water quality, geology, paleomagnetism, and stratigraphy of the eastern Snake River Plain and Idaho National Laboratory, Idaho.—Continued

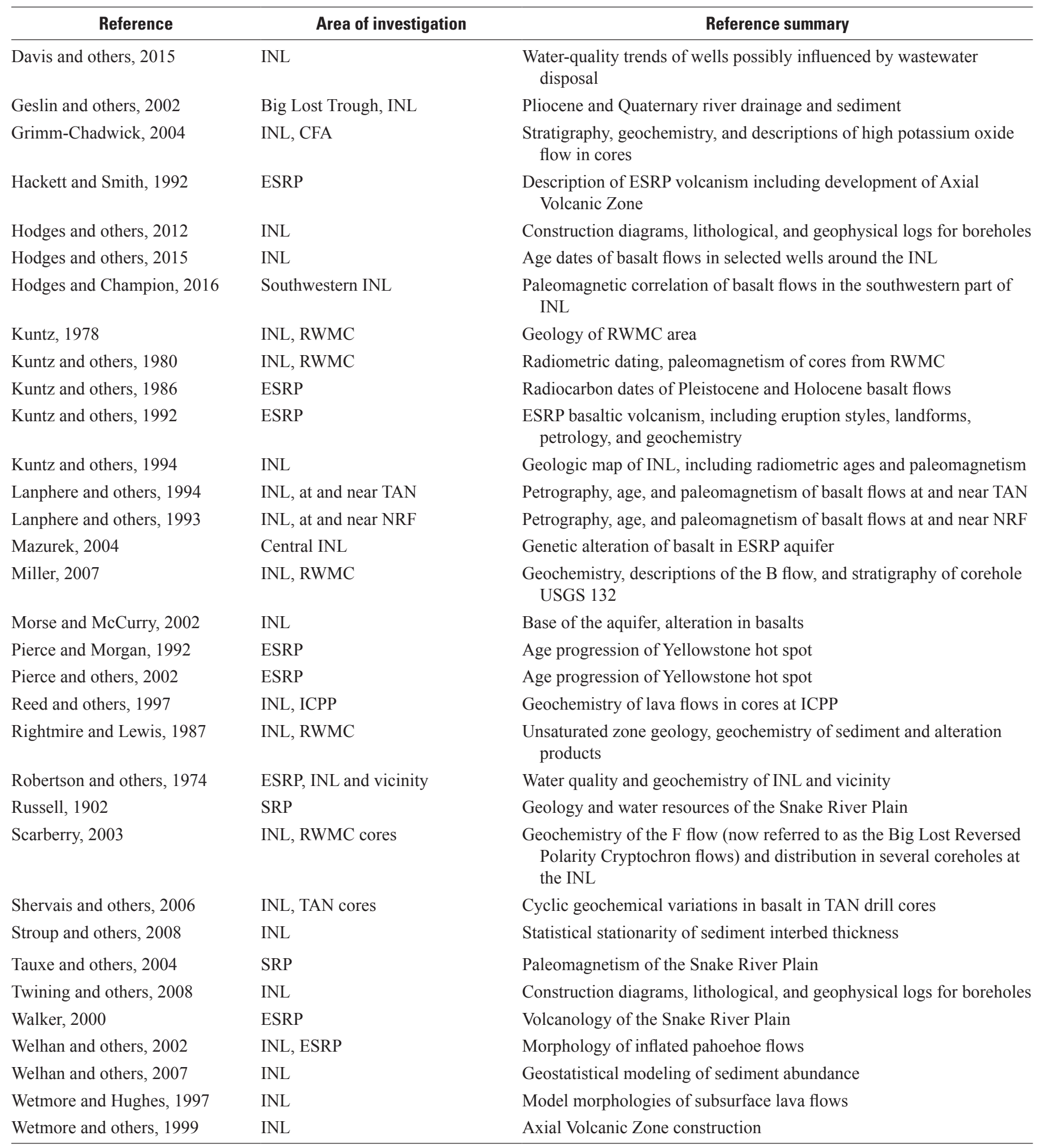




\section{Geohydrologic Setting}

The INL is located on the west-central part of the ESRP. The ESRP developed when the North American tectonic plate moved southwestward over a fixed upper mantlemelting anomaly beginning about 17 million years ago (Pierce and Morgan, 1992; Pierce and others, 2002; Morgan and McIntosh, 2005). Thermal disruption resulted in a time transgressive series of silicic volcanic fields, characterized by positive geoid anomalies, rhyolitic resurgent caldera eruptions, emplacement of a mid-crustal mafic sill, and subsidence with later basaltic plains magmatism (Braile and others, 1982; Anders and Sleep, 1992; Peng and Humphries, 1998; Rodgers and others, 2002; Shervais and others, 2006). The part of the ESRP now occupied by the INL was the site of resurgent caldera activity, including the Picabo volcanic field from $10.2 \pm 0.06$ million years ago (Ma) to $7.9 \pm 0.4 \mathrm{Ma}$ (Kellogg and others, 1994; McCurry and Hughes, 2006), and the Heise volcanic field from $7.05 \pm 0.04 \mathrm{Ma}$ to $4.43 \pm 0.08 \mathrm{Ma}$ (Pierce and Morgan, 1992; Pierce and others, 2002; Morgan and McIntosh, 2005; McCurry and Hughes, 2006).

The ESRP is subsiding in the wake of the Yellowstone hot spot calderas (Braile and others, 1982; Anders and Sleep, 1992; McQuarrie and Rodgers, 1998; Rodgers and others, 2002). The ESRP subsided as it was filled, first with silicic material from the caldera eruptions, then later with tholeiitic basalt, and to a minor degree, with fluvial sediments washed out onto the ESRP (Blair, 2002; Bestland and others, 2002; Geslin and others, 2002). The total volume of basalt filling the ESRP is estimated to be $9,600 \mathrm{mi}^{3}$ (Kuntz, 1992).

The ESRP is an example of basaltic plains volcanism (Greeley, 1982). This form of basaltic volcanism is intermediate in style between flood basalts, such as the Columbia River Basalt Group, and shield volcano eruptions, such as those in Hawaii and Iceland. Basaltic eruptions on the ESRP generated a land surface formed from coalesced shield volcanoes that produced voluminous tube-fed pahoehoe flows and fissure eruptions (Greeley, 1982). Basaltic plains volcanism is characterized by relatively low effusion rates, long recurrence intervals, low total volumes of lava erupted, and the prevalence of monogenetic volcanoes (Kuntz, 1992). ESRP shield volcanoes produced flows that ranged from about 3 to $131 \mathrm{ft}$ thick. The extent of some ESRP flows may be as large as $155 \mathrm{mi}^{2}$. The ESRP olivine tholeiite basalt flows may be as much as $22 \mathrm{mi}$ long, and the accumulated volume of a shield volcano may be as large as $1.7 \mathrm{mi}^{3}$ (Kuntz and others, 1992). The flank areas of typical ESRP low shield volcanoes have slopes of less than 1 degree, and summit and vent areas have slopes of approximately 5 degrees (Greeley, 1982).

Large, old vents are sometimes preserved as hills surrounded by basalt flows from younger vents; a good example is AEC Butte (fig. 1; Kuntz and others, 1994; Kuntz and others, 2003; Kuntz and others, 2007; Skipp and others, 2009).

More than 95 percent of the total volume of basalt in the ESRP is composed of tube-fed pahoehoe flows erupted from monogenetic shield volcanoes and lava cones (Kuntz and others, 1992). Basaltic lava fields, partially mantled with loess, cover the ESRP. The greatest numbers of eruptive centers are in the Axial Volcanic Zone ([AVZ]; fig. 1; Hackett and Smith, 1992). The AVZ is a constructional volcanic highland that parallels the long axis of the ESRP (Hackett and Smith, 1992; Kuntz and others, 1992, 1994; Anderson and Liszewski, 1997; Anderson, Ackerman, and others, 1996; Anderson and others, 1999; Hughes and others, 1999; Wetmore and others, 1999).

Basaltic eruptions have occurred on the ESRP about every 32,000-140,000 years over the entire INL (Champion and others, 2002). Eruptions in the northern part of the INL occur at longer intervals, and the shortest recurrence interval eruptions occur on or near the axis of the ESRP in the AVZ. Accumulation rates are highest in and around the AVZ (fig. 1; Champion and others, 2002). Most basalt flows and vents on the land surface in the southern part of the INL have normal magnetic polarity. These basalt flows and vents were erupted during the Brunhes Normal Polarity Chron and are less than $0.78 \mathrm{Ma}$. Some surface basalt flows and vents in the northern part of the INL have reversed magnetic polarity and erupted during the Matuyama Reversed Polarity Chron (2.581-0.78 Ma) (fig. 3; Ogg and Smith, 2004).

The basaltic flows and interbedded sediment combine to form the ESRP aquifer, which is one of the most productive aquifers in the United States (U.S. Geological Survey, 1985, p. 193). Water in the aquifer generally moves from northeast to southwest and eventually discharges to springs along the Snake River downstream of Twin Falls, Idaho, about $100 \mathrm{mi}$ southwest of the INL. Water moves horizontally through basalt interflow zones and vertically through joints and interfingering edges of interflow zones. Infiltration of surface water, heavy pumpage, geologic conditions, and seasonal fluxes in recharge and discharge locally affect the movement of groundwater (Garabedian, 1986). The ESRP aquifer is recharged primarily from infiltration of applied irrigation water, infiltration of streamflow, groundwater inflow from adjoining mountain drainage basins, and infiltration of precipitation.

At the INL, depth to water in wells completed in the ESRP aquifer ranges from about $200 \mathrm{ft}$ in the northern part of the site to more than $900 \mathrm{ft}$ in the southeastern part. A significant proportion of the groundwater moves through the upper 200-800 ft of basaltic rocks (Mann, 1986, p. 21). Ackerman (1991, p. 30) and Bartholomay and others (1997, table 3 ) reported a range of transmissivity of basalt in the upper part of the aquifer of 1.1-760,000 $\mathrm{ft}^{2} / \mathrm{d}$. The hydraulic gradient at the INL ranges from 2 to $10 \mathrm{ft} / \mathrm{mi}$, with an average of $4 \mathrm{ft} / \mathrm{mi}$ (Bartholomay and others, 2017, fig. 9). Horizontal flow velocities of about $2-26 \mathrm{ft} / \mathrm{d}$ have been calculated based on the movement of various constituents in different areas of the aquifer at and near the INL (Robertson and others, 1974; Mann and Beasley, 1994; Cecil and others, 2000; Plummer and others, 2000; Busenberg and others, 2001). These flow rates equate to a travel time of between 55 and 700 years for water beneath the INL to travel to springs that discharge at the terminus of the ESRP aquifer. Localized tracer tests at the INL have revealed vertical- and horizontal-transport rates as high as $60-150 \mathrm{ft} / \mathrm{d}$ (Nimmo and others, 2002; Duke and others, 2007). 
Geomagnetic Time Scale

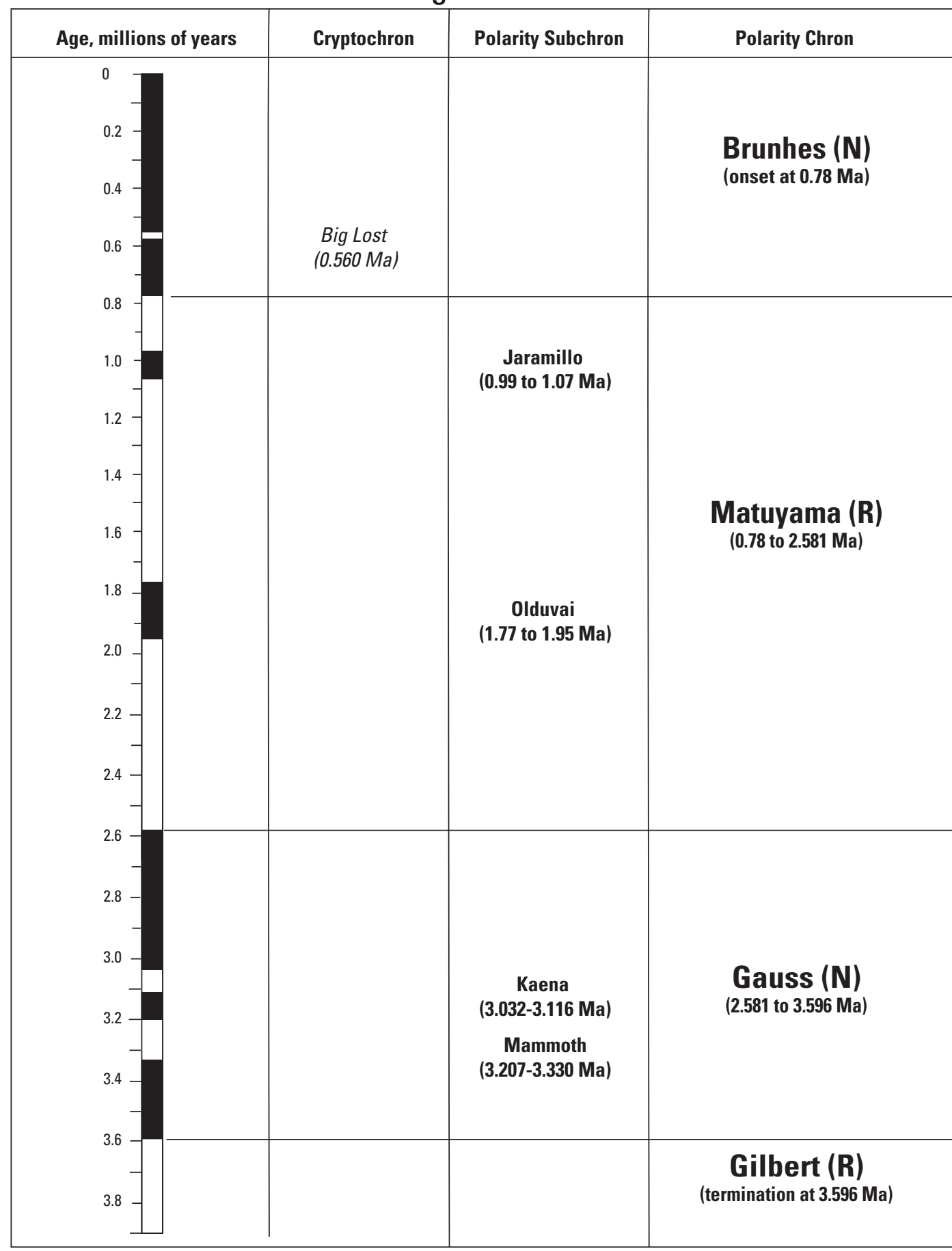

Figure 3. Geomagnetic time scale. Modified from Champion and others (1988), with new data from Ogg and Smith (2004).
EXPLANATION

Normal magnetic polarity

Reversed magnetic polarity

Ma Million years 


\section{Sampling and Analytical Techniques for Basalt Flows}

The drill cores used for this study were carefully logged and sampled using INL Lithologic Core Storage Library protocols described in Davis and others (1997). Prior to sampling, the core material was described and the tops and bottoms of lava flows and flow units were identified. A lava flow unit is defined herein as the minimum subdivision of a lava flow, possessing quenched bottom and top surfaces, and typically part of a nearly contemporaneous group of other lava flow units. Basalt flow names used in this report were defined in more detail in Champion and others (2011) and Hodges and Champion (2016) and included labeling conventions, geomagnetic framework, sample collection, and correlation techniques. The basalt flow identified as being present in each well in the interval that the water samples are collected are given in table 2. Depths for basalt flows were measured by tape in feet and tenths of a foot from known marks recorded on wooden plugs or footage marked on the cores in the core boxes at the drill site. The wooden plugs are placed, or the drillers make marks on cores at the time of coring; the recorded measured depths are logged at the end of each core run. Descriptions of cores used to define the basalt flows in the aquifer are given in appendix A. Stratigraphy for wells used for wastewater determinations that did not have a core were interpreted from the nearest core to the well. Several wells at and downgradient of the ATR Complex and INTEC (see Bartholomay and others, 2017, fig. 6, for more information on wells not included) were not included in table 2 as they would have been determined to have the same stratigraphy because of similar distance to wells with known stratigraphy. The wells not included had open intervals (Bartholomay and others, 2017, table 2) similar to the wells used in this study and would not have contributed additional information to the study.

\section{Sampling and Analytical Techniques for Tritium Concentrations}

The tritium data used to determine whether or not wastewater influenced well water concentrations are from previously published data and includes data from the USGS National Water Information System database (accessible at https://maps.waterdata.usgs.gov/mapper/) (table 3). Tritium data from 11 multilevel monitoring well systems (MLMS) at the INL are reported in Bartholomay and Twining (2010) and Bartholomay and others (2015). The water samples collected from the MLMS were analyzed at the USGS Menlo Park Research Laboratory using electronic enrichment and liquid scintillation techniques to obtain lower detection levels (2.2 picocuries per liter $[\mathrm{pCi} / \mathrm{L}]$ ) than other data used for this study. Other tritium data used for this study were from wells completed in the upper part of the ESRP aquifer and were analyzed by the DOE Radiological and Environmental Sciences Laboratory with detection levels of about $200 \mathrm{pCi} / \mathrm{L}$. Sampling methods and collection for water samples are described in Bartholomay and others (2014). Concentrations less than the detection level are labeled not detected (ND) in figure 4 . The analytical results for tritium are presented with calculated analytical uncertainties (counting errors) in table 3. The probability is about 67 percent that the true radionuclide concentration is in a range of the reported concentrations plus or minus the uncertainty. Tritium data are compared to the estimated lower level of background $(75 \mathrm{pCi} / \mathrm{L})$ determined by Orr and others (1991) even though a more recent background level as reported by Bartholomay and Hall (2016) is about $35 \mathrm{pCi} / \mathrm{L}$ for groundwater in the southwestern part of the INL. The larger background is used to account for older water that would not have gone through as many half-life decay series as water samples collected more recently, because the data used for wastewater determination were collected between 1991 and 2016 (table 3). Chloride data also are listed in table 3 because chloride is another constituent commonly disposed of in wastewater at the ATR Complex and INTEC. Chloride readily moves in the aquifer system and is used to identify the presence or absence of wastewater in some of the wells with tritium concentrations less than the $200 \mathrm{pCi} / \mathrm{L}$ detection level. 


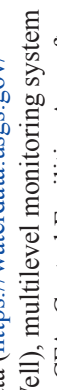

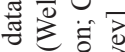

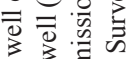

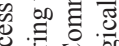

政的

列

岛

응

讙

䠉要

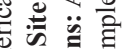

实禹记

$\stackrel{2}{2}$

岁文至

垔这

$\because \frac{0}{0}$

国吾令

ดे

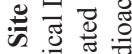

\begin{tabular}{|c|c|c|c|c|c|c|c|c|c|c|c|c|c|c|c|c|c|c|}
\hline \multicolumn{2}{|c|}{ 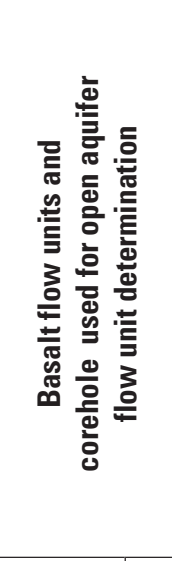 } & 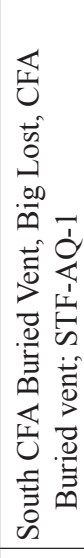 & 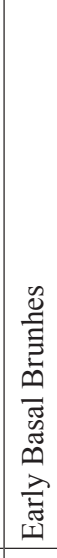 & 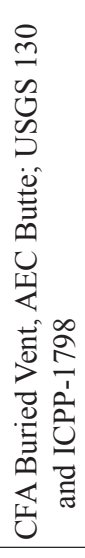 & 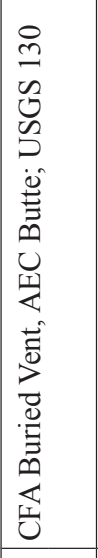 & 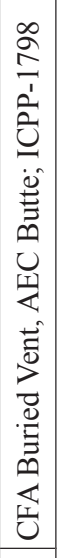 & 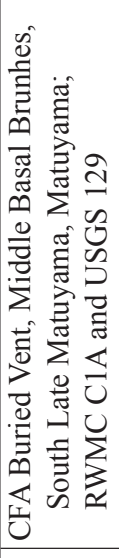 & & 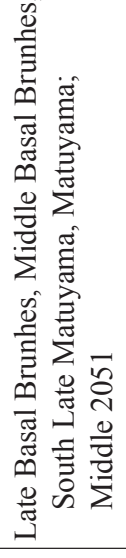 & 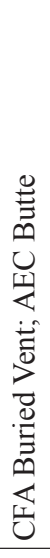 & 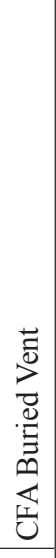 & 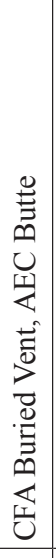 & 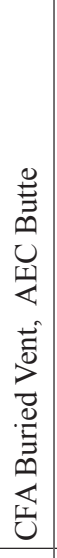 & 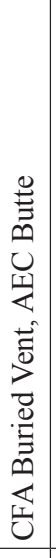 & 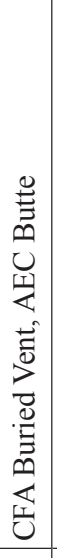 & 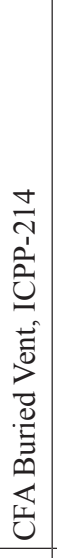 & 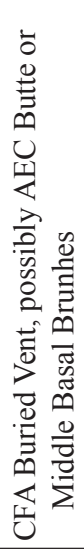 & 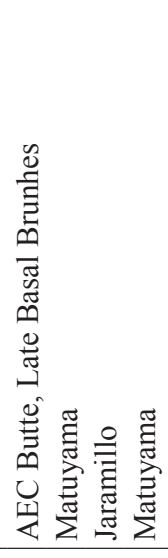 \\
\hline \multirow{2}{*}{ 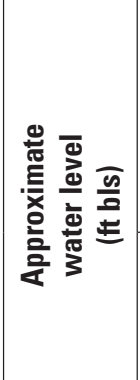 } & 总 & 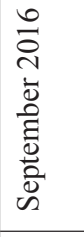 & $\begin{array}{l}0 \\
0 \\
0 \\
\frac{1}{0} \\
\frac{2}{2}\end{array}$ & 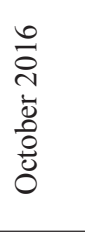 & 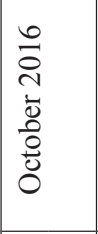 & $\begin{array}{l}0 \\
\cdots \\
\stackrel{1}{1} \\
0\end{array}$ & 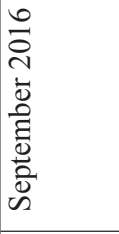 & & $\begin{array}{l}0 \\
\stackrel{0}{\sim} \\
0 \\
\Xi \\
\Xi\end{array}$ & 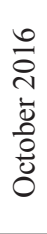 & 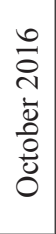 & 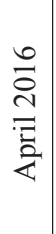 & 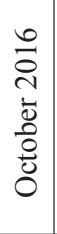 & 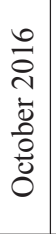 & 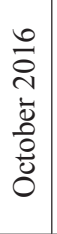 & 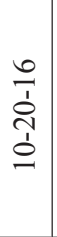 & $\begin{array}{l}0 \\
\frac{1}{1} \\
\text { ஸे } \\
0\end{array}$ & \\
\hline & 垔 & $\begin{array}{l}\infty \\
\infty \\
+\infty\end{array}$ & in & $\stackrel{\leftrightarrow}{\stackrel{q}{q}}$ & $\begin{array}{l}\infty \\
\stackrel{\infty}{+}\end{array}$ & $\begin{array}{l}\vec{\sim} \\
\stackrel{a}{q}\end{array}$ & $\hat{8}$ & & 串 & $\begin{array}{l}n \\
b\end{array}$ & 苟 & 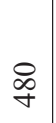 & ஓे & $\stackrel{\substack{\infty \\
+}}{ }$ & $\stackrel{2}{\circ}$ & $\begin{array}{l}\infty \\
\infty \\
i \\
i\end{array}$ & $\begin{array}{l}\infty \\
\infty \\
\infty \\
\infty\end{array}$ & \\
\hline \multicolumn{2}{|c|}{ 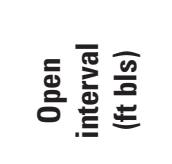 } & 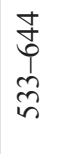 & $\overleftrightarrow{Z}$ & 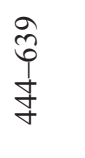 & 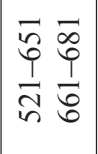 & & $\begin{array}{l}0 \\
\frac{1}{1} \\
8 \\
0\end{array}$ & 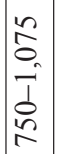 & $\begin{array}{l}0 \\
\substack{n \\
0 \\
0 \\
0}\end{array}$ & $\overleftrightarrow{Z}$ & $\overleftrightarrow{z}$ & $\overleftrightarrow{Z}$ & $\overleftrightarrow{z}$ & $\overleftrightarrow{z}$ & $\begin{array}{l}\approx \\
\vdots \\
\vdots \\
\infty \\
\infty \\
+\end{array}$ & $\begin{array}{l}\hat{i} \\
i \\
\frac{1}{\infty} \\
\infty\end{array}$ & $\begin{array}{l}\text { हे } \\
\hat{b} \\
\dot{b}\end{array}$ & 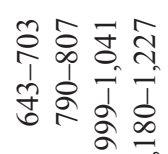 \\
\hline \multicolumn{2}{|c|}{ 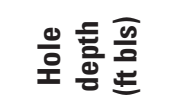 } & $\underset{\delta}{\mathbb{Z}}$ & $\stackrel{\infty}{\circ}$ & $\mathscr{\infty}$ & $\vec{\infty}$ & $\underset{\infty}{\infty}$ & $\stackrel{n}{0}$ & & $\stackrel{2}{n}$ & & $\stackrel{\infty}{\stackrel{q}{\sigma}}$ & $\stackrel{\infty}{0}$ & $\hat{\mathscr{b}}$ & $\begin{array}{l}\infty \\
d\end{array}$ & 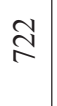 & in & 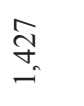 & \\
\hline \multicolumn{2}{|c|}{ 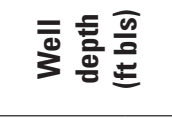 } & 过 & $\mathbb{z}$ & ठે & $\vec{\infty}$ & $\frac{0}{1}$ & $\stackrel{n}{0}$ & & $\stackrel{8}{n}$ & $\overleftrightarrow{z}$ & $\overleftrightarrow{z}$ & $\overleftrightarrow{z}$ & $\overleftrightarrow{z}$ & $\overleftrightarrow{z}$ & $\mathbb{z}$ & $\hat{\imath}$ & 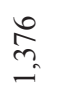 & \\
\hline \multicolumn{2}{|c|}{ 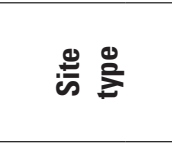 } & $\overline{\overline{0}}$ & $\begin{array}{l}0 \\
\frac{0}{0} \\
\overline{0} \\
0\end{array}$ & $\overline{\overline{0}}$ & $\overline{\overline{0}}$ & $\overline{\bar{c}}$ & $\overline{\overline{0}}$ & & $\overline{\overline{0}}$ & 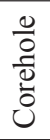 & 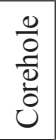 & $\begin{array}{l}\frac{0}{0} \\
\frac{0}{0} \\
0 \\
0\end{array}$ & $\begin{array}{l}0 \\
0 \\
0 \\
0 \\
0 \\
0 \\
0\end{array}$ & 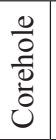 & $\begin{array}{l}\frac{0}{0} \\
0 \\
0 \\
0 \\
0\end{array}$ & $\overline{\overline{0}}$ & $\sum_{\Sigma}^{\infty}$ & \\
\hline \multicolumn{2}{|c|}{ 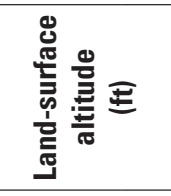 } & $\begin{array}{l}\tilde{\sigma} \\
\curvearrowright \\
\sigma\end{array}$ & & $\begin{array}{l}\infty \\
\widetilde{+} \\
\dot{+}\end{array}$ & $\begin{array}{l}\bar{\sigma} \\
\text { }\end{array}$ & $\begin{array}{l}\tilde{\sigma} \\
\tilde{\sigma}\end{array}$ & \begin{tabular}{l}
\multirow{\Delta}{\Delta}{} \\
in
\end{tabular} & & $\begin{array}{l}\vec{\infty} \\
\stackrel{\sigma}{\sigma}\end{array}$ & & & & & & $\begin{array}{l}\overrightarrow{\tilde{\sigma}} \\
\vec{\sigma}\end{array}$ & $\begin{array}{l}\stackrel{\circ}{2} \\
\stackrel{\sigma}{\sigma}\end{array}$ & $\begin{array}{l}\stackrel{\infty}{\sim} \\
\stackrel{\sigma}{\sigma}\end{array}$ & \\
\hline \multicolumn{2}{|c|}{ 总 } & 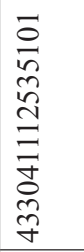 & $\begin{array}{l}\overrightarrow{0} \\
\frac{0}{n} \\
\frac{\pi}{0} \\
0 \\
\frac{\pi}{0} \\
0 \\
0 \\
0 \\
z\end{array}$ & 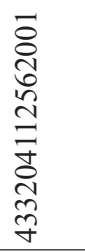 & 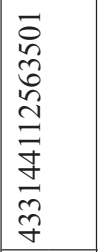 & 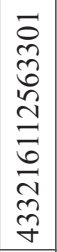 & 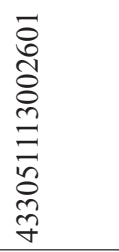 & & 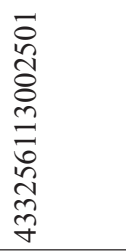 & $\begin{array}{l}\overrightarrow{0} \\
\frac{0}{n} \\
: \overrightarrow{0} \\
0 \\
\frac{\pi}{0} \\
0 \\
0 \\
0 \\
z\end{array}$ & 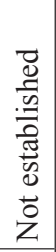 & 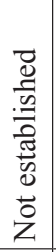 & 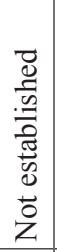 & 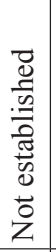 & 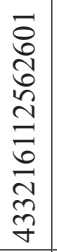 & 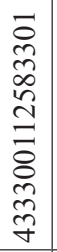 & 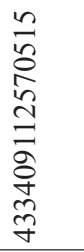 & 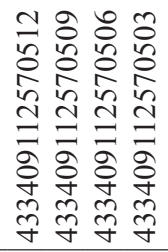 \\
\hline \multicolumn{2}{|l|}{ 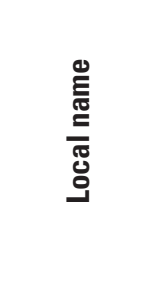 } & 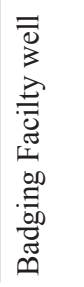 & 志 & 丞 & 点 & 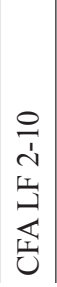 & 亥 & & 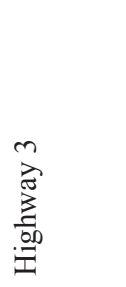 & 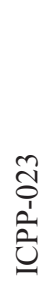 & 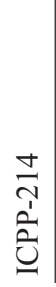 & 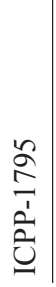 & $\begin{array}{l}2 \\
\stackrel{2}{1} \\
\stackrel{1}{0} \\
0\end{array}$ & 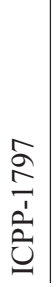 & 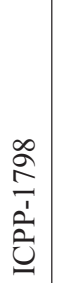 & 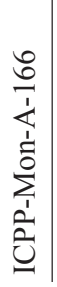 & \multicolumn{2}{|c|}{ 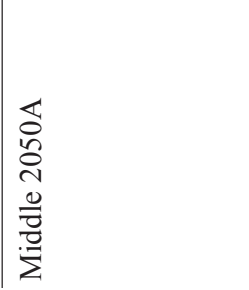 } \\
\hline
\end{tabular}




\begin{tabular}{|c|c|c|c|c|c|c|c|c|c|c|c|c|c|c|c|c|c|}
\hline \multicolumn{2}{|c|}{ 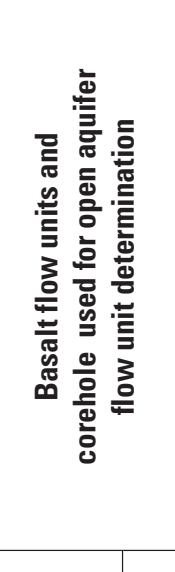 } & 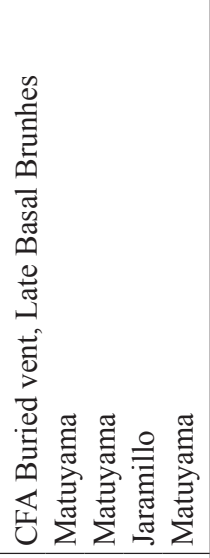 & 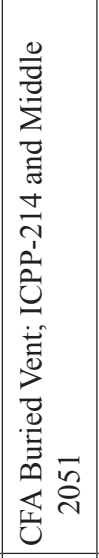 & 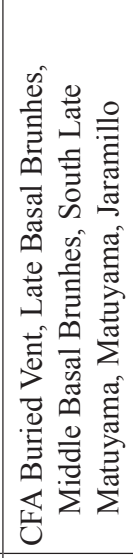 & 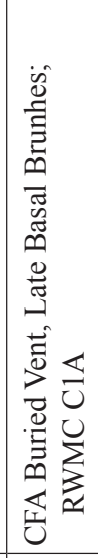 & 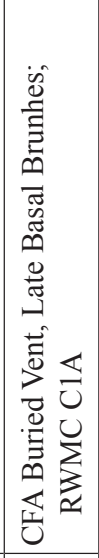 & 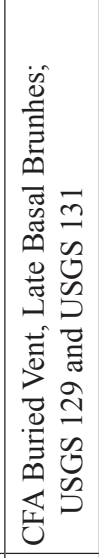 & 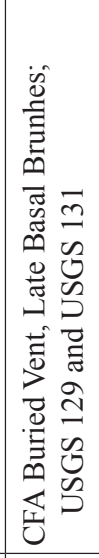 & 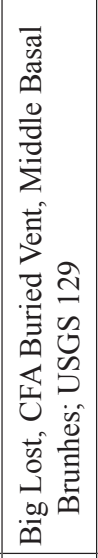 & 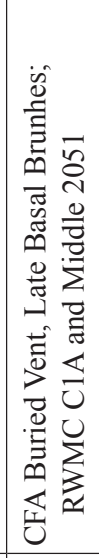 & 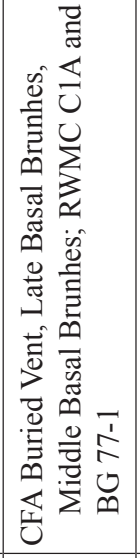 & 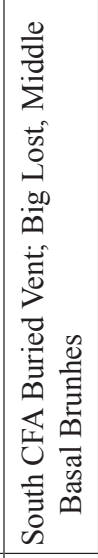 & 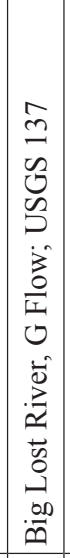 & 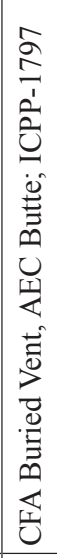 & 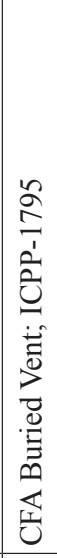 & 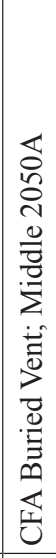 & 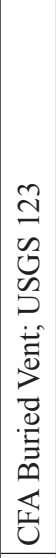 \\
\hline \multirow{2}{*}{ 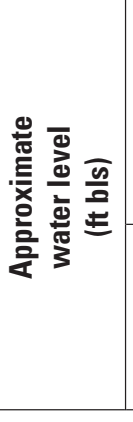 } & 总 & $\begin{array}{l}0 \\
\frac{1}{1} \\
0 \\
1 \\
0 \\
0\end{array}$ & 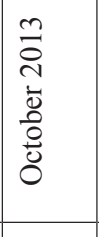 & 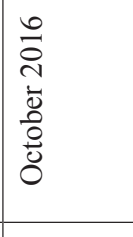 & $\begin{array}{l}0 \\
\frac{1}{2} \\
\frac{1}{1} \\
0\end{array}$ & $\begin{array}{c}0 \\
1 \\
\stackrel{1}{1} \\
0 \\
\end{array}$ & $\frac{0}{1}$ & 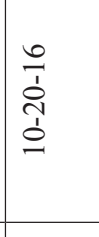 & $\begin{array}{l}\frac{0}{1} \\
\frac{1}{1} \\
\frac{1}{2}\end{array}$ & 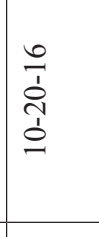 & 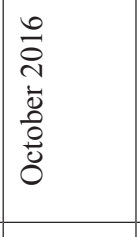 & 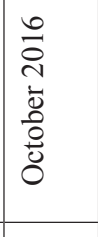 & $\mid \begin{array}{c}0 \\
\frac{1}{1} \\
\frac{1}{1} \\
-\end{array}$ & $\begin{array}{l}0 \\
0 \\
1 \\
0 \\
0 \\
0 \\
-1\end{array}$ & $\begin{array}{l}0 \\
0 \\
1 \\
0 \\
0 \\
0 \\
0\end{array}$ & $\frac{0}{\frac{1}{2}}$ & $\begin{array}{l}0 \\
1 \\
1 \\
0 \\
0 \\
0\end{array}$ \\
\hline & 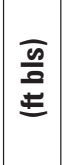 & $\begin{array}{l}n \\
0 \\
0 \\
0 \\
n\end{array}$ & $\frac{m}{n}$ & 命 & $\begin{array}{l}\overrightarrow{6} \\
\stackrel{0}{0} \\
\text { in }\end{array}$ & $\begin{array}{l}n \\
a \\
\dot{\infty} \\
n\end{array}$ & $\begin{array}{l}2 \\
\text { ì } \\
\text { in }\end{array}$ & $\begin{array}{l}\hat{b} \\
\dot{+} \\
\dot{n}\end{array}$ & $\frac{n}{1}$ & $\stackrel{?}{\frac{i}{6}}$ & $\hat{n}$ & $\underset{\infty}{\infty}$ & $\mid \begin{array}{c}\dot{d} \\
\dot{d} \\
\dot{\sigma}\end{array}$ & 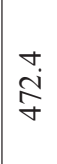 & $\begin{array}{c}n \\
\tilde{n} \\
n \\
\infty \\
\dot{\infty}\end{array}$ & 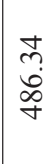 & $\frac{n}{8}$ \\
\hline \multicolumn{2}{|c|}{ 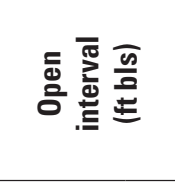 } & 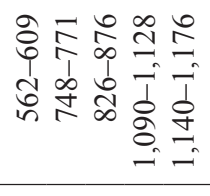 & $\begin{array}{l}0 \\
0 \\
0 \\
0 \\
0 \\
0\end{array}$ & $\overleftrightarrow{z}$ & $\begin{array}{l}\hat{⿵} \\
\hat{1} \\
\hat{\delta}\end{array}$ & $\begin{array}{l}\infty \\
0 \\
0 \\
\infty \\
\infty \\
i n\end{array}$ & $\begin{array}{l}\text { ते } \\
0 \\
2 \\
\tilde{n}\end{array}$ & $\begin{array}{l}\mathbb{N} \\
\hat{n} \\
\infty \\
i n\end{array}$ & $\begin{array}{l}\text { qut } \\
0 \\
\text { ô } \\
\text { in }\end{array}$ & $\begin{array}{l}n \\
0 \\
0 \\
0 \\
\infty \\
n\end{array}$ & $\begin{array}{l}n \\
0 \\
0 \\
0 \\
0 \\
0 \\
i n\end{array}$ & $\begin{array}{l}\infty \\
\infty \\
\infty \\
\infty \\
n \\
n\end{array}$ & $\mid \begin{array}{l}\infty \\
0 \\
0 \\
\infty \\
0 \\
0\end{array}$ & $\mid \begin{array}{l}\infty \\
0 \\
0 \\
1 \\
6 \\
+ \\
+1\end{array}$ & $\begin{array}{l}2 \\
\hat{n} \\
1 \\
0 \\
i n\end{array}$ & $\frac{a}{q}$ & 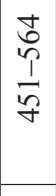 \\
\hline \multicolumn{2}{|c|}{ 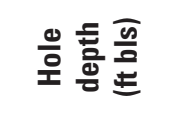 } & $\stackrel{2}{=}$ & స్ర & $\begin{array}{l}n \\
\infty \\
\infty \\
-1\end{array}$ & 8 & $\infty$ & ర్ర & $\begin{array}{l}\infty \\
\infty \\
i n\end{array}$ & to & ? & $\ddot{\infty}$ & $\frac{m}{7}$ & $\begin{array}{l}t \\
6 \\
6\end{array}$ & $\frac{0}{6}$ & $\stackrel{n}{n}$ & $\underset{n}{\mathbb{n}}$ & $\frac{1}{6}$ \\
\hline \multicolumn{2}{|c|}{ 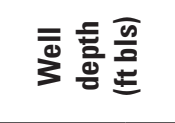 } & $\stackrel{n}{=}$ & గ్రి & $\overleftrightarrow{z}$ & $\tilde{\sigma}$ & 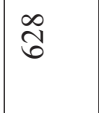 & $\underset{\widetilde{b}}{\mathbb{b}}$ & $\underset{i n}{\mathbb{N}}$ & fơ & $\hat{\widehat{\sigma}}$ & $\ddot{\infty}$ & $\begin{array}{l}\infty \\
i \\
i n\end{array}$ & $\begin{array}{l}t \\
6\end{array}$ & 文 & $\underset{n}{\mathbb{n}}$ & $\widetilde{g}$ & 总 \\
\hline \multicolumn{2}{|c|}{$\stackrel{\Xi}{\dot{s}}$} & $\sum_{\Sigma}^{\infty}$ & $\overline{\overline{0}}$ & \begin{tabular}{|l|}
$\frac{0}{0}$ \\
$\frac{0}{0}$ \\
0 \\
0
\end{tabular} & $\frac{\overline{0}}{3}$ & $\overline{0}$ & $\overline{\overline{0}}$ & $\overline{\overline{0}}$ & $\overline{\overline{0}}$ & $\overline{\overline{0}}$ & $\overline{\overline{0}}$ & 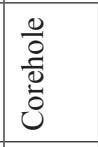 & $\overline{0}$ & $\overline{\overline{0}}$ & $\overline{\overline{0}}$ & $\overline{\overline{0}}$ & $\overline{0}$ \\
\hline \multicolumn{2}{|c|}{ 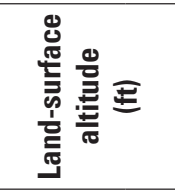 } & $\begin{array}{l}\hat{\alpha} \\
\hat{\sigma}\end{array}$ & $\begin{array}{l}\bar{E} \\
\dot{0} \\
+\end{array}$ & $\begin{array}{l}2 \\
0 \\
0 \\
i n\end{array}$ & $\begin{array}{l}0 \\
0 \\
0 \\
i n\end{array}$ & $\begin{array}{l}n \\
o \\
i n\end{array}$ & $\begin{array}{l}+ \\
\sigma \\
\sigma \\
\sigma\end{array}$ & $\begin{array}{l}n \\
\hat{a} \\
\sigma\end{array}$ & $\begin{array}{l}\hat{\sigma} \\
\text { in }\end{array}$ & $\begin{array}{l}0 \\
\text { है } \\
\text { in }\end{array}$ & $\begin{array}{l}n \\
8 \\
n\end{array}$ & $\begin{array}{l}\vec{F} \\
\dot{\sigma}\end{array}$ & $\begin{array}{c}0 \\
\tilde{\delta} \\
i n\end{array}$ & $\begin{array}{l}\frac{n}{2} \\
\frac{\sigma}{\sigma}\end{array}$ & $\begin{array}{l}\text { ふे } \\
\text { +े }\end{array}$ & $\begin{array}{l}\bar{\sigma} \\
\hat{\sigma}\end{array}$ & $\begin{array}{l}n \\
\frac{n}{\alpha} \\
\dot{f}\end{array}$ \\
\hline \multicolumn{2}{|c|}{ 亪 } & 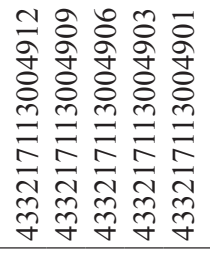 & 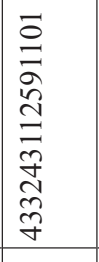 & 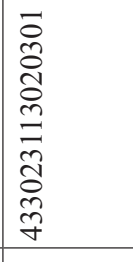 & 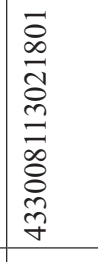 & 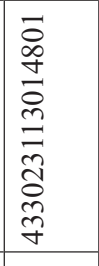 & 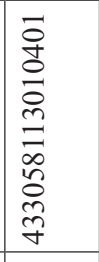 & 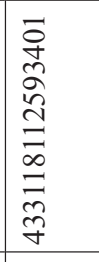 & 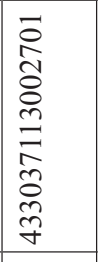 & 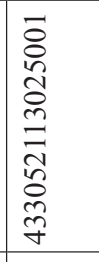 & 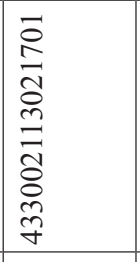 & 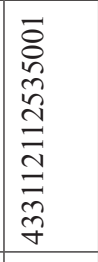 & 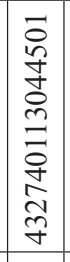 & 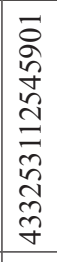 & 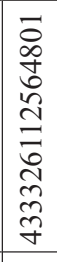 & 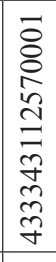 & 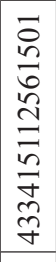 \\
\hline \multicolumn{2}{|l|}{ 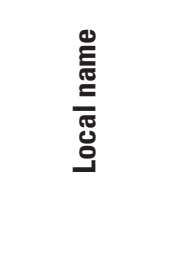 } & 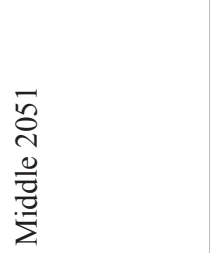 & 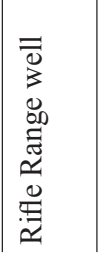 & 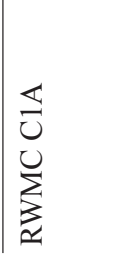 & $\sum_{\substack{\infty \\
\infty}}^{\infty}$ & $\sum_{\substack{n \\
0}}^{\infty}$ & $\sum_{\substack{\infty \\
\sum}}^{\infty}$ & 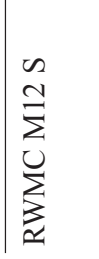 & $\sum_{\substack{\infty \\
\infty}}^{\infty}$ & $\sum_{\substack{\infty \\
\sum}}^{\infty}$ & $\begin{array}{l}0 \\
0 \\
0 \\
0 \\
0 \\
\sum_{2}^{2}\end{array}$ & 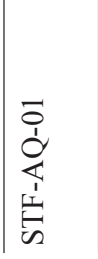 & $\left|\begin{array}{l}a \\
0 \\
0 \\
0\end{array}\right|$ & $\begin{array}{l}0 \\
\text { 1 } \\
0 \\
0 \\
0 \\
1\end{array}$ & $\begin{array}{l}n \\
n \\
n \\
0 \\
0 \\
D\end{array}$ & $\begin{array}{l}0 \\
\text { n } \\
n \\
0 \\
0 \\
0\end{array}$ & $\begin{array}{l}q \\
f \\
y \\
0 \\
0 \\
D\end{array}$ \\
\hline
\end{tabular}




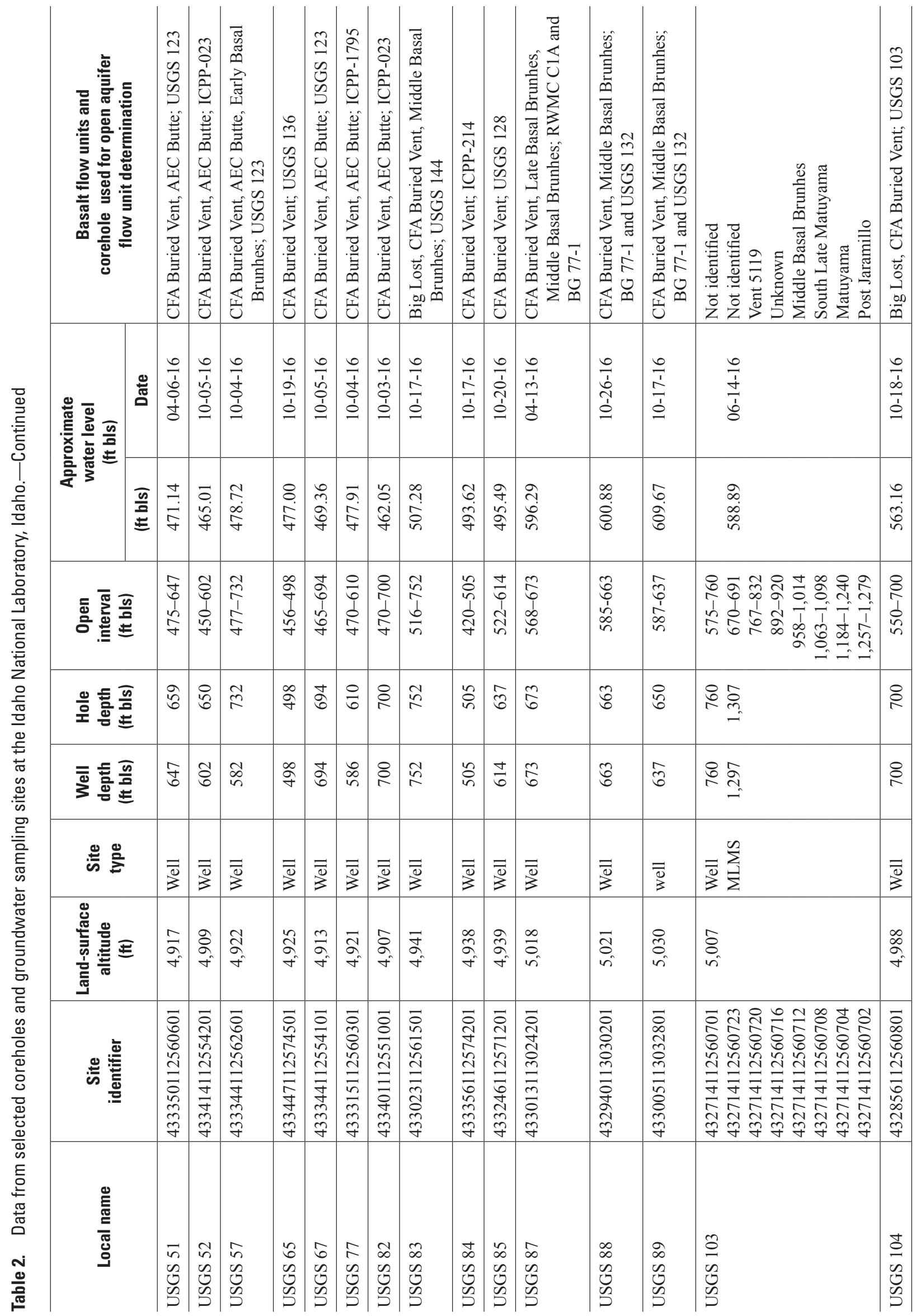




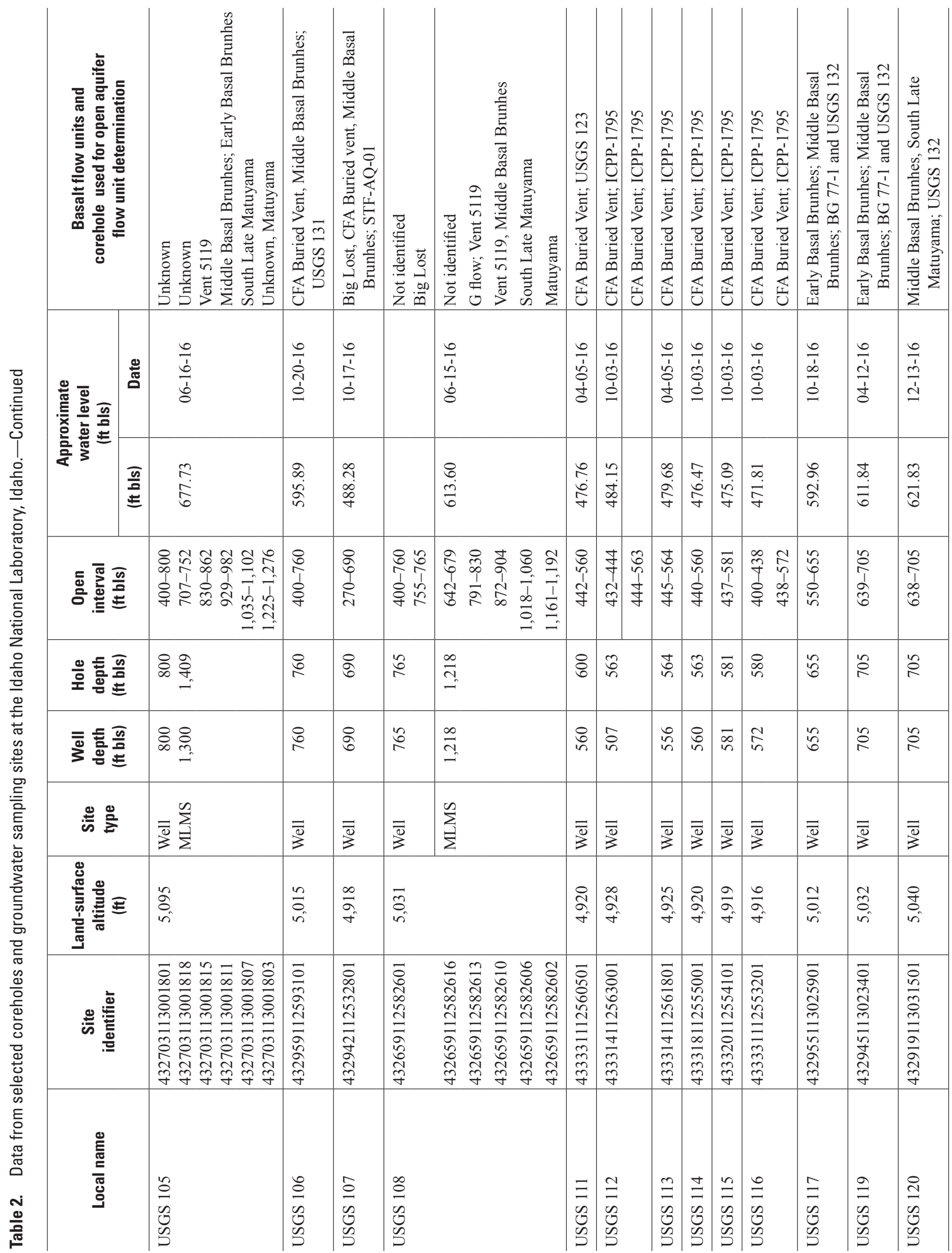




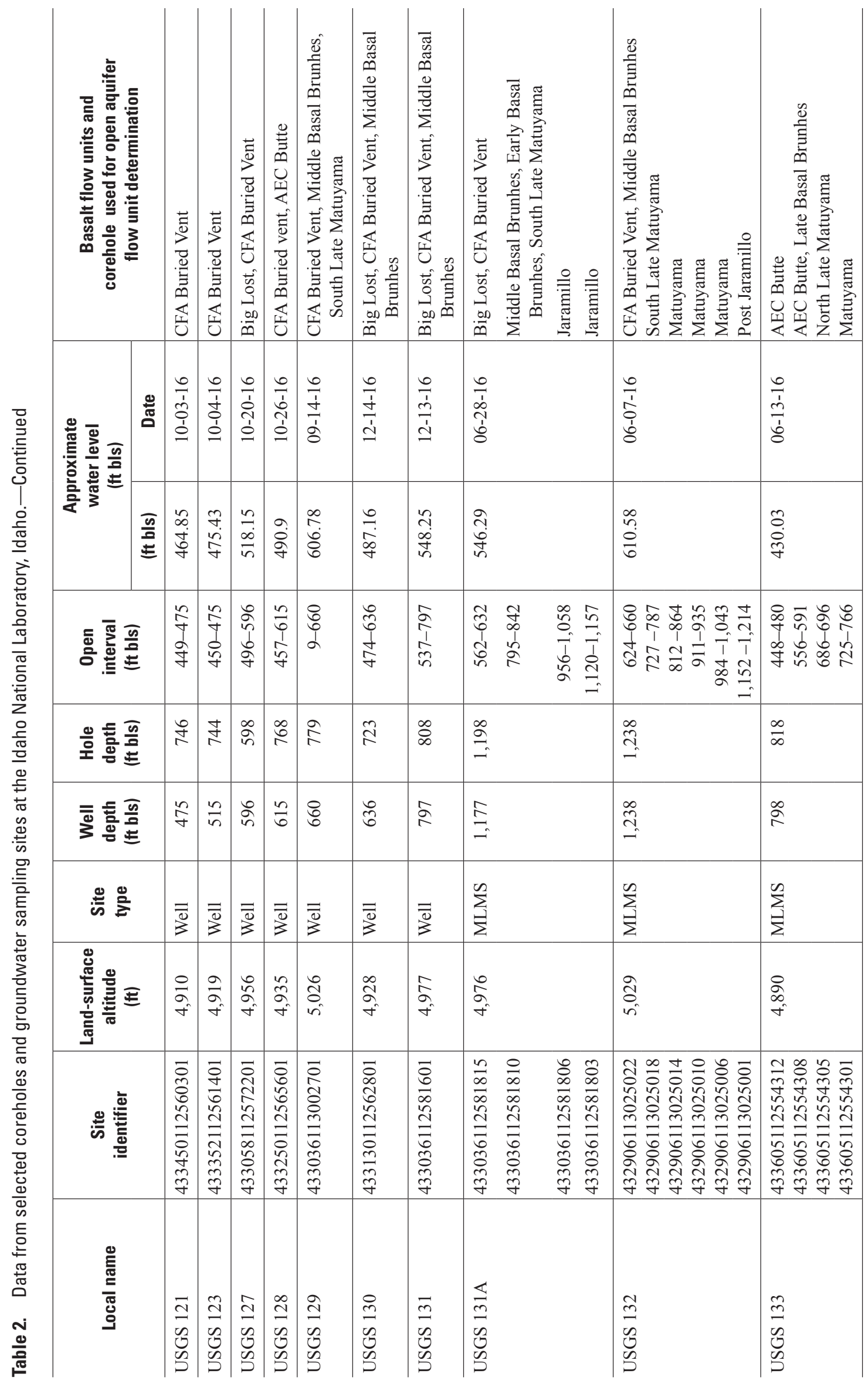




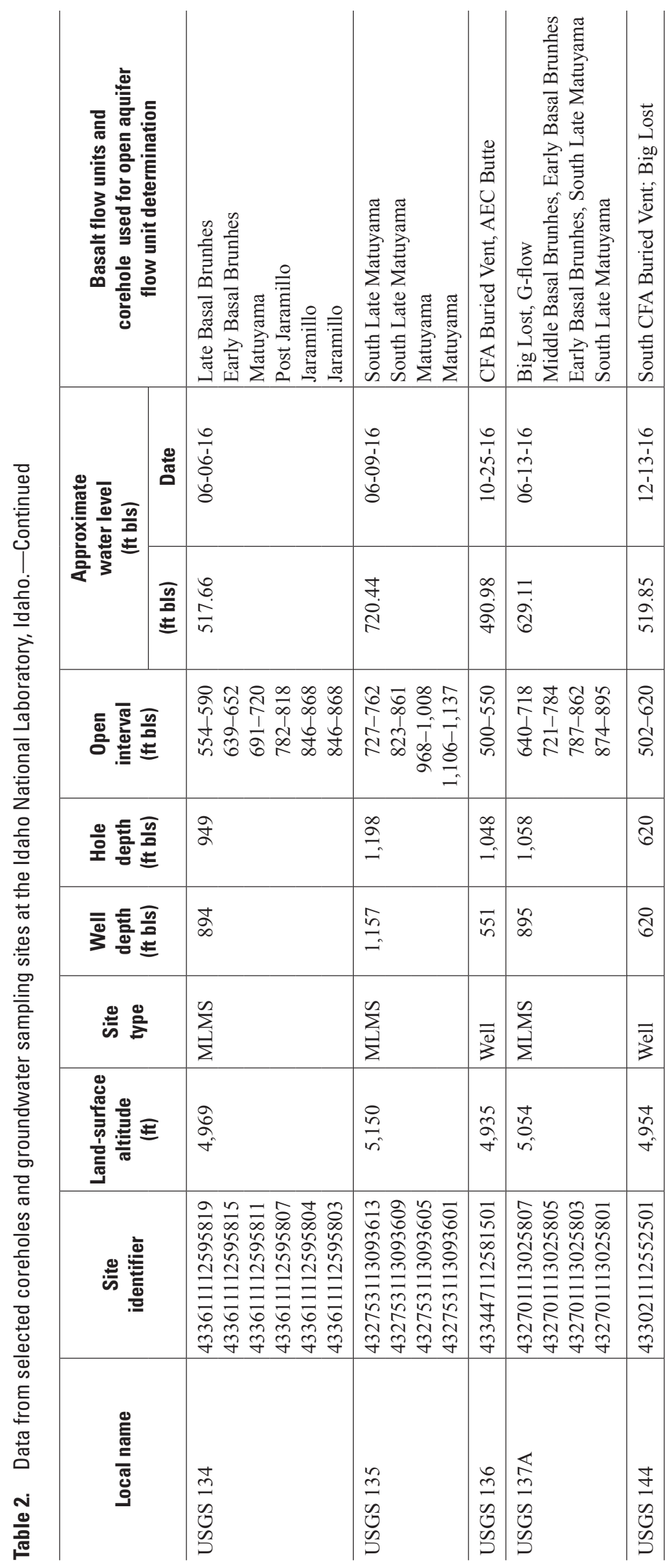




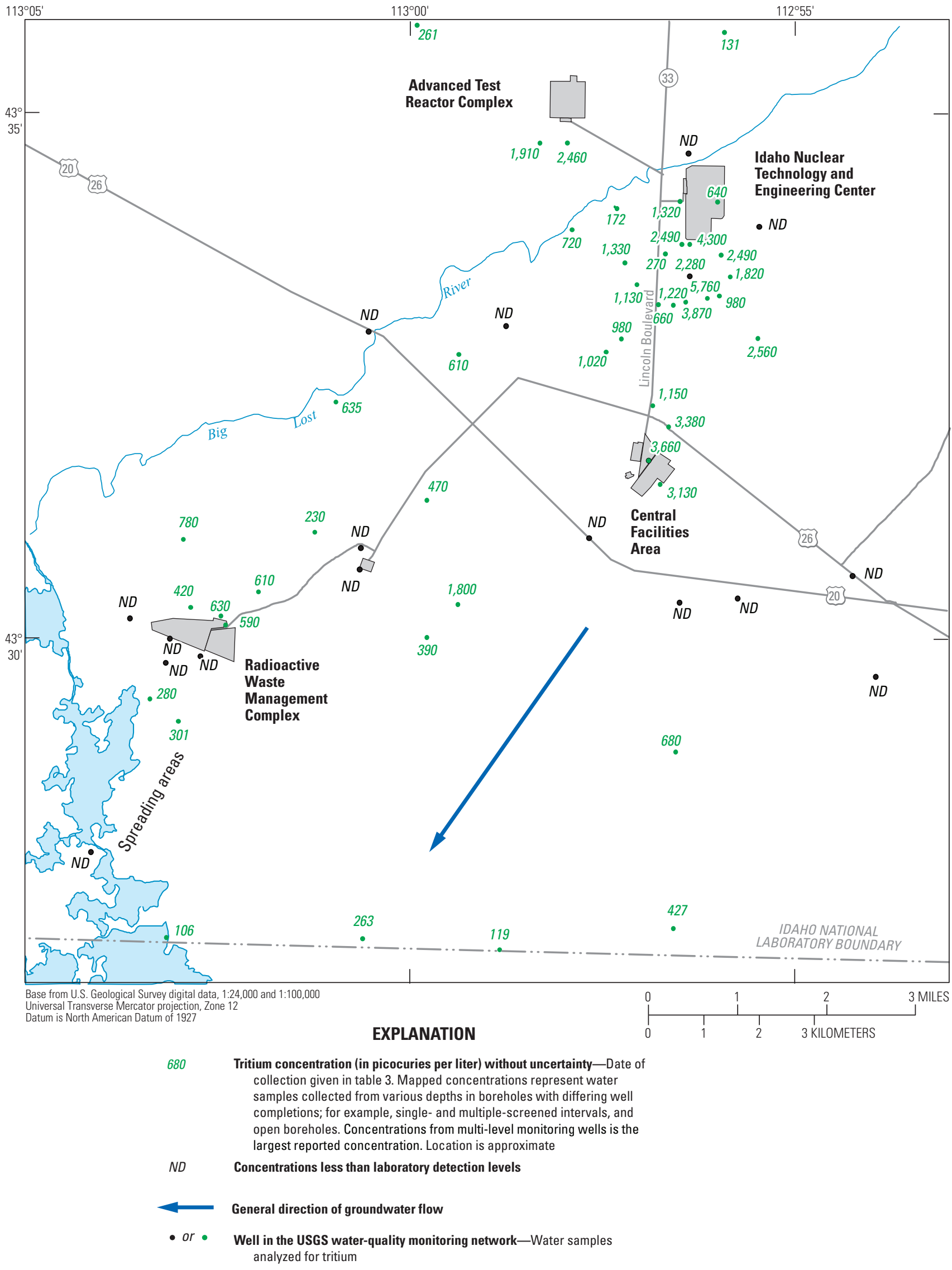

Figure 4. Tritium concentrations in water sampled from wells in the southwestern part of the Idaho National Laboratory, Idaho. See figure 2 for well numbers. 
Table 3. Hydraulic conductivity, tritium, and chloride data for selected groundwater sampling sites at the Idaho National Laboratory, Idaho.

[Locations of wells are shown in figures 1 and 2. Hydraulic conductivity values are from Anderson and others (1999, table 2). Value for upper zones of multilevel wells USGS 103, 105, 108, and 137A are for open intervals in wells before multi-level systems were installed. USGS 109 is used for the upper level of USGS 137A because wells are at the same location. Local name: Local well identifier used in this study. Site identifier: Unique numerical identifier used to access well data (https://waterdata.usgs.gov/nwis). Open interval: For multi-level systems, the open interval is a hydraulically isolated depth interval. Abbreviations: $\mathrm{ft}$ bls, foot below land surface; $\mathrm{ft} / \mathrm{d}$, foot per day; $\mathrm{mg} / \mathrm{L}$, milligram per liter; NA, not available; $\mathrm{pCi} / \mathrm{L}$, picocuries per liter; >, greater than; \pm , plus or minus]

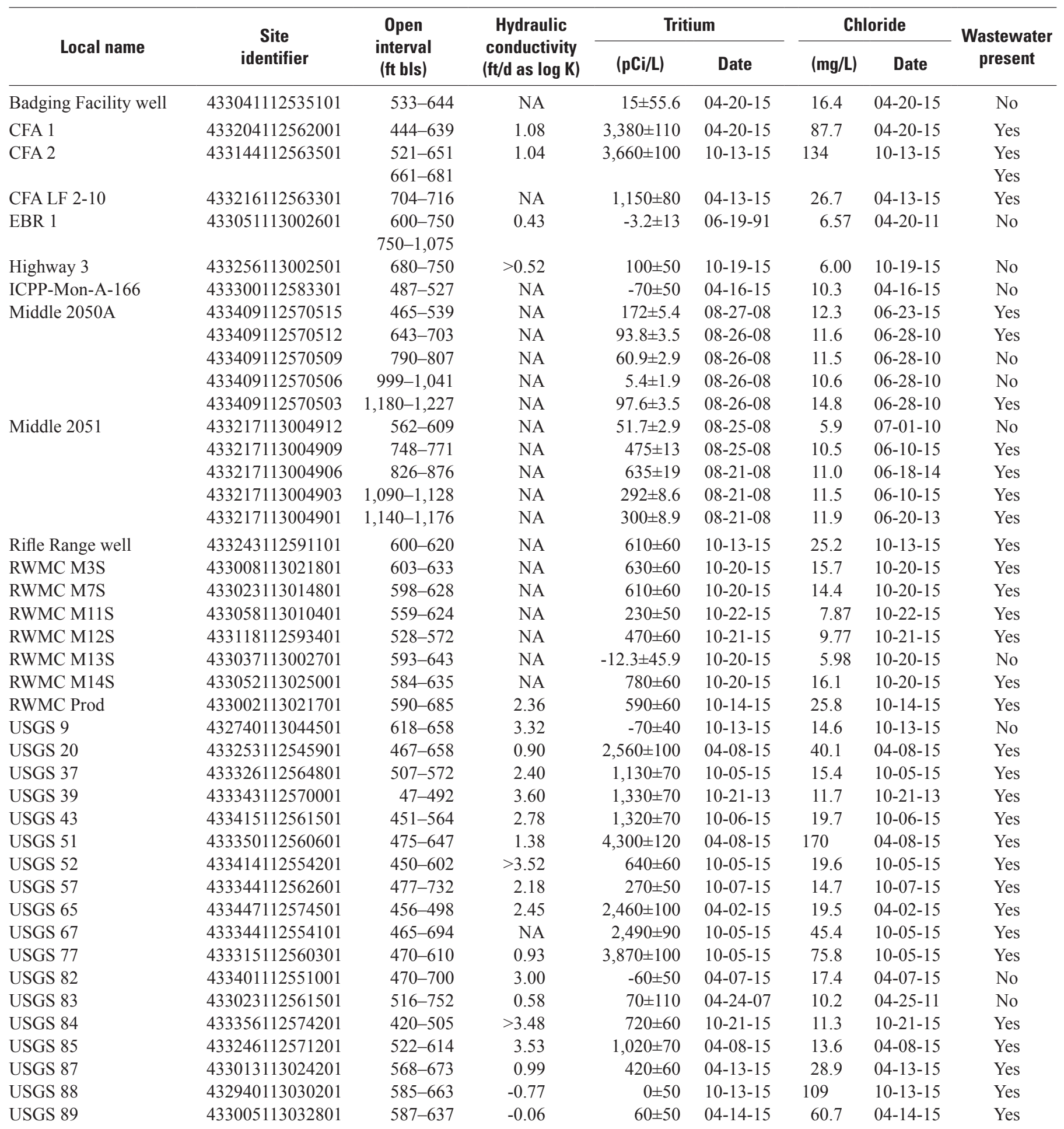


Table 3. Hydraulic conductivity, tritium, and chloride data for selected groundwater sampling sites at the Idaho National Laboratory, Idaho.-Continued

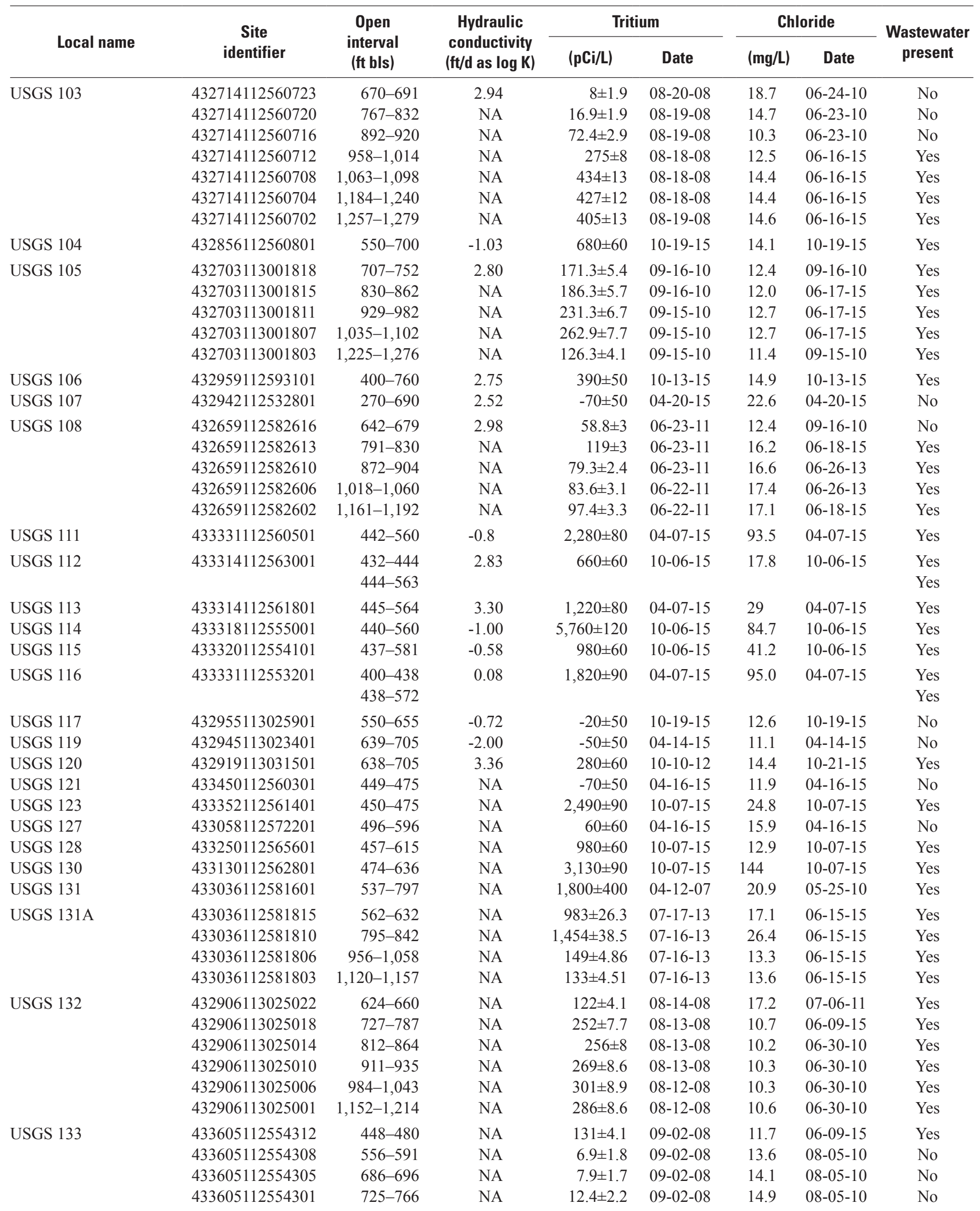


Table 3. Hydraulic conductivity, tritium, and chloride data for selected groundwater sampling sites at the Idaho National Laboratory, Idaho.-Continued

\begin{tabular}{|c|c|c|c|c|c|c|c|c|}
\hline \multirow{2}{*}{ Local name } & \multirow{2}{*}{$\begin{array}{c}\text { Site } \\
\text { identifier }\end{array}$} & \multirow{2}{*}{$\begin{array}{c}\text { Open } \\
\text { interval } \\
\text { (ft bls) }\end{array}$} & \multirow{2}{*}{$\begin{array}{c}\text { Hydraulic } \\
\text { conductivity } \\
\text { (ft/d as log K) }\end{array}$} & \multicolumn{2}{|c|}{ Tritium } & \multicolumn{2}{|c|}{ Chloride } & \multirow{2}{*}{$\begin{array}{c}\text { Wastewater } \\
\text { present }\end{array}$} \\
\hline & & & & (pCi/L) & Date & $(\mathrm{mg} / \mathrm{L})$ & Date & \\
\hline \multirow[t]{5}{*}{ USGS 134} & 433611112595819 & $554-590$ & NA & $17 \pm 1.9$ & 09-04-08 & 9.47 & $06-08-15$ & No \\
\hline & 433611112595815 & $639-652$ & NA & $261 \pm 7.3$ & 09-04-08 & 9.7 & $06-29-11$ & Yes \\
\hline & 433611112595811 & $691-720$ & NA & $9.1 \pm 1.7$ & 09-03-08 & 7.28 & $07-10-13$ & No \\
\hline & 433611112595807 & $782-818$ & NA & $3 \pm 1.8$ & 09-03-08 & 10.3 & $06-21-10$ & No \\
\hline & 433611112595803 & $846-868$ & NA & $88.7 \pm 3.2$ & 09-03-08 & 8.4 & $06-21-10$ & No \\
\hline USGS 136 & 433447112581501 & $500-550$ & 2.4 & $1,910 \pm 100$ & $10-25-11$ & 14.0 & $10-15-15$ & Yes \\
\hline \multirow[t]{4}{*}{ USGS 137A } & 432701113025807 & $640-718$ & 2.78 & $106 \pm 3.83$ & $07-15-13$ & 13.7 & $06-22-15$ & Yes \\
\hline & 432701113025805 & $721-784$ & NA & $89.2 \pm 3.47$ & $07-15-13$ & 12.1 & $06-22-15$ & Yes \\
\hline & 432701113025803 & $787-862$ & NA & $67.6 \pm 2.97$ & $07-15-13$ & 11.1 & $06-22-15$ & No \\
\hline & 432701113025801 & $874-895$ & NA & $64.8 \pm 2.87$ & $07-15-13$ & 11.1 & $06-22-15$ & No \\
\hline USGS 144 & 433021112552501 & $502-620$ & NA & $80 \pm 60$ & $12-07-16$ & 17.8 & $12-07-16$ & No \\
\hline
\end{tabular}

Correlation between Basalt Flows and Wastewater Constituents in the Aquifer

Recent mapping of basalt flows at the INL using paleomagnetic inclination data (Champion and others, 2011; 2013; Hodges and Champion, 2016) has led to a better understanding and correlation of stratigraphic sequences at the INL. The extent of basalt flow units from youngest (Big Lost flow, fig. 5A) to oldest (Matuyama flow, fig. 5H) are shown in figure 5 . The stratigraphic sequence of the flows from youngest to oldest is shown in table 4, which lists the percentage of wells that show wastewater influence compared with the wells with flows that do not show wastewater influence.

Table 4. Stratigraphic sequence and percentage of flows influenced by wastewater at the Idaho National Laboratory, Idaho.

[Basalt flow units were defined in Champion and others (2011) and Hodges and Champion (2016) and are arranged from youngest to oldest. The sequence for CFA Buried vent and G flow, AEC Butte and Vent 5119, North and South Late Matuyama, and the unknown flow in USGS 103, and Late Basal Brunhes are uncertain because these flows are not present together in any well to determine overlap and age dates that are available indicate that the flows are about the same age]

\begin{tabular}{|c|c|c|c|c|}
\hline \multirow[b]{2}{*}{ Basalt flow unit } & \multicolumn{3}{|c|}{ Number of wells } & \multirow{2}{*}{$\begin{array}{c}\text { Wastewater } \\
\text { influence } \\
\text { (percent) }\end{array}$} \\
\hline & $\begin{array}{c}\text { Flow } \\
\text { identified }\end{array}$ & $\begin{array}{c}\text { Wastewater } \\
\text { detected }\end{array}$ & $\begin{array}{l}\text { Wastewater } \\
\text { not detected }\end{array}$ & \\
\hline South CFA Buried Vents & 2 & 0 & 2 & 0 \\
\hline Big Lost & 13 & 5 & 8 & 38 \\
\hline CFA Buried Vent & 52 & 43 & 9 & 83 \\
\hline G flow & 2 & 2 & 2 & 100 \\
\hline Vent 5119 & 3 & 2 & 1 & 67 \\
\hline AEC Butte & 14 & 14 & 0 & 100 \\
\hline Late Basal Brunhes & 12 & 7 & 5 & 58 \\
\hline Unknown flow in USGS 103 & 1 & 0 & 1 & 0 \\
\hline Middle Basal Brunhes & 20 & 11 & 9 & 55 \\
\hline Early Basal Brunhes & 7 & 5 & 2 & 71 \\
\hline North Late Matuyama & 1 & 0 & 1 & 0 \\
\hline South Late Matuyama & 10 & 7 & 3 & 70 \\
\hline Unknown flow in USGS 105 & 1 & 1 & 1 & 100 \\
\hline Matuyama & 11 & 5 & 6 & 45 \\
\hline Post Jaramillo & 2 & 1 & 1 & 50 \\
\hline Jaramillo & 4 & 3 & 1 & 75 \\
\hline
\end{tabular}




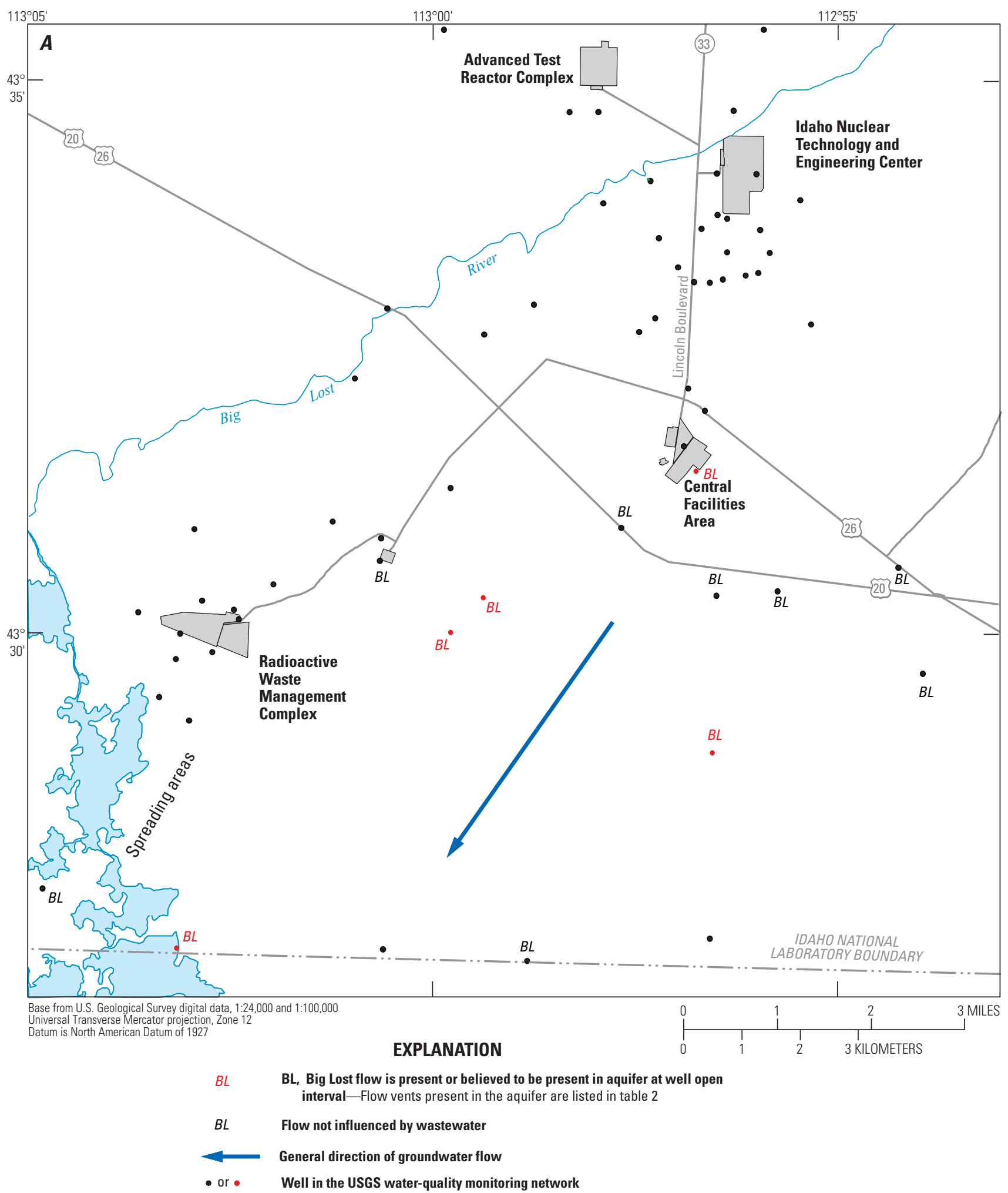

Figure 5. Occurrence of basalt flows $(A)$ Big Lost, $(B)$ CFA Buried Vent, $(C)$ AEC Butte, $(D)$ Late Basal Brunhes, $(E)$ Middle Basal Brunhes, ( $F$ Early Basal Brunhes, $(G)$ South Late Matuyama, and $(H)$ Matuyama in wells in the southwestern part of the Idaho National Laboratory, Idaho. See figure 2 for well numbers. 


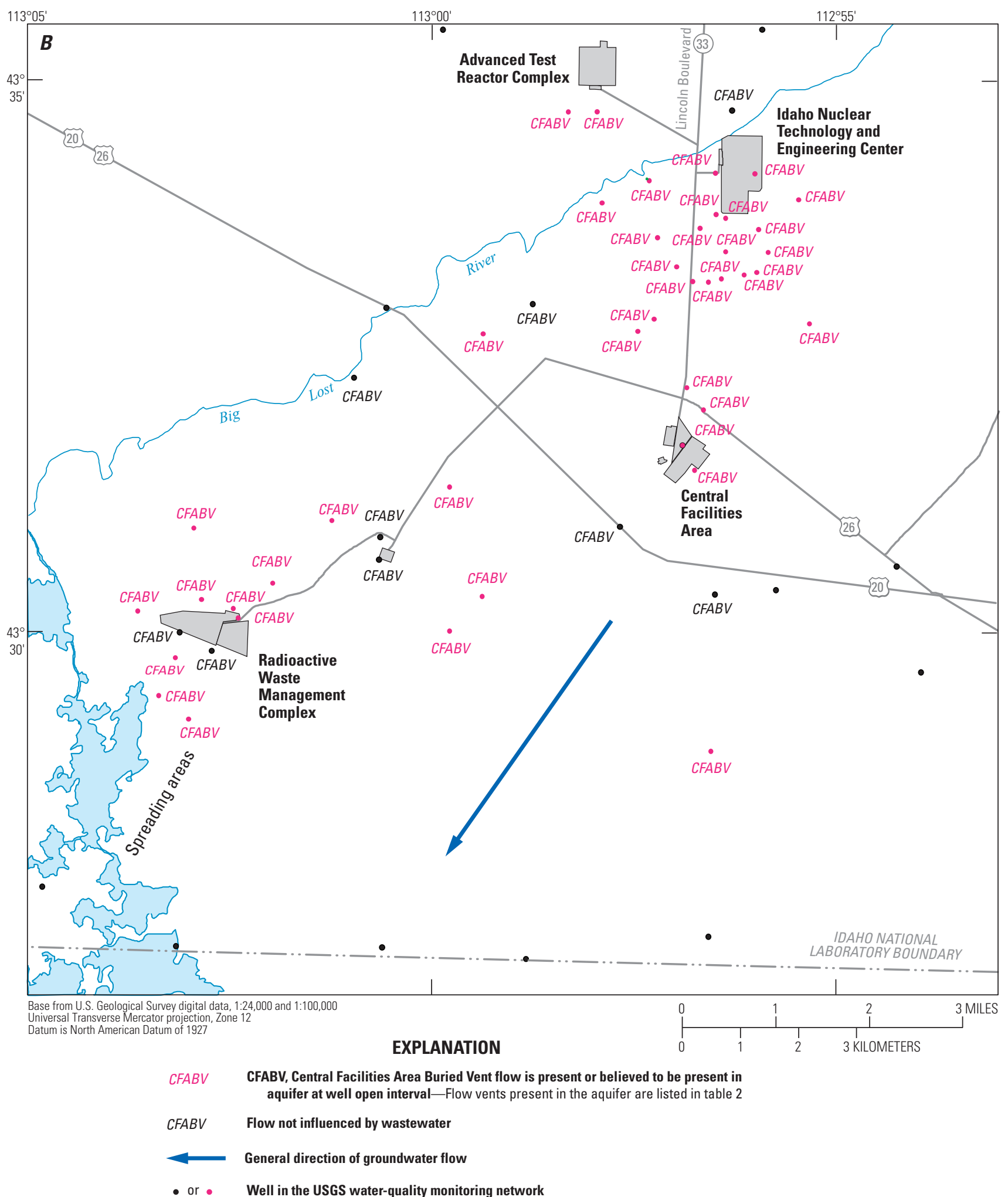

Figure 5.-Continued 


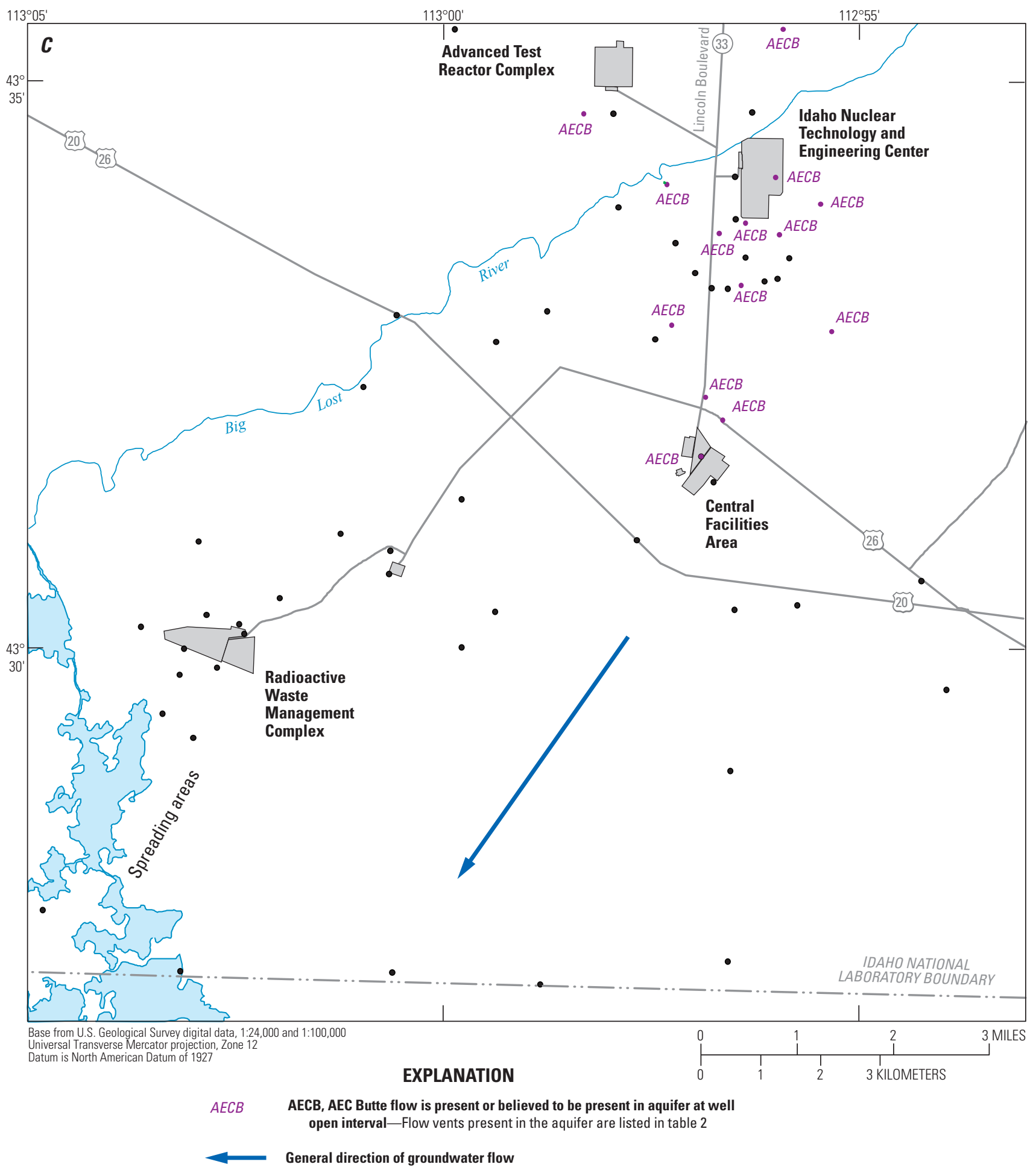

- or Well in the USGS water-quality monitoring network.

Figure 5.-Continued 


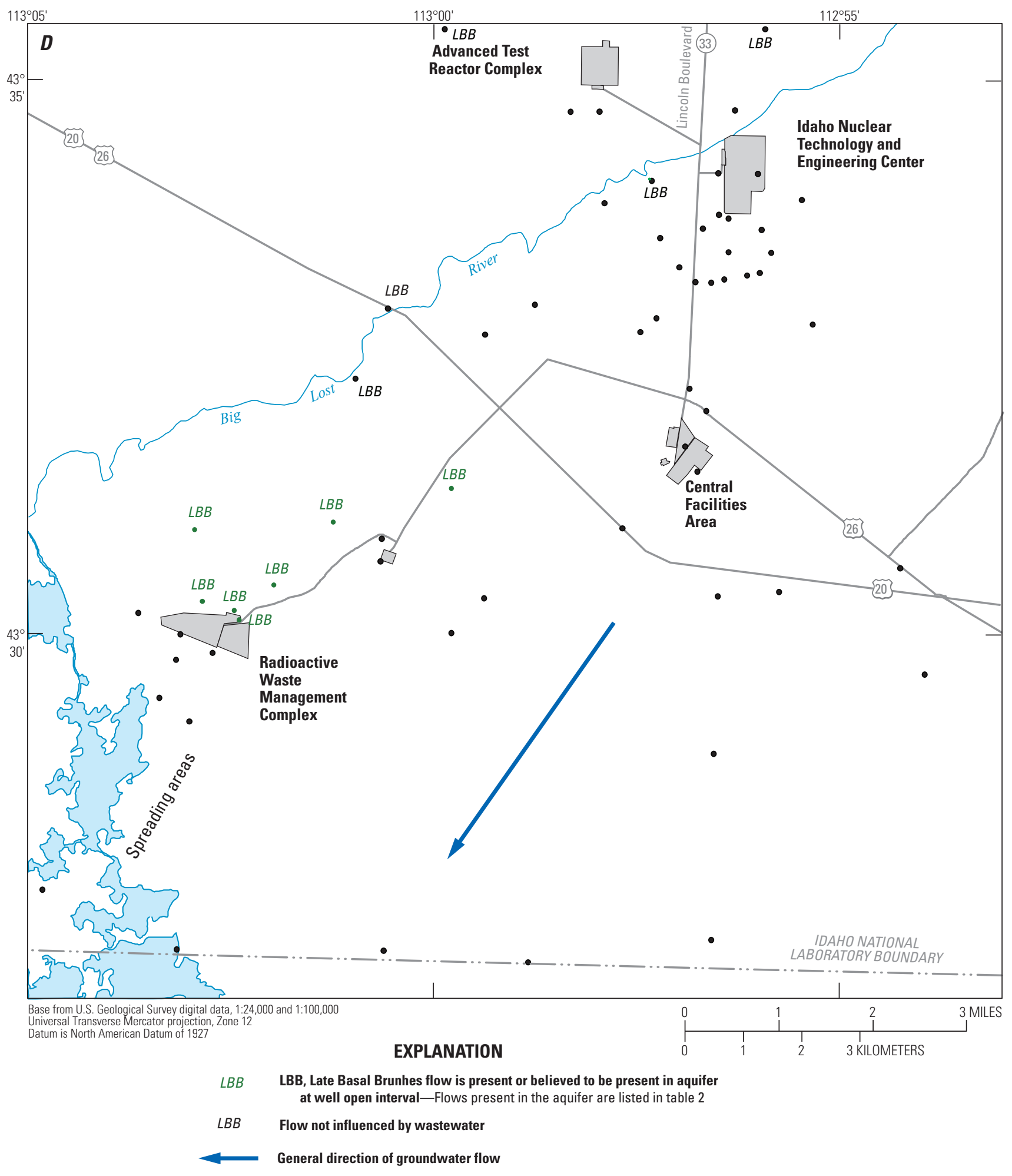

- or Well in the USGS water-quality monitoring network

Figure 5.-Continued 


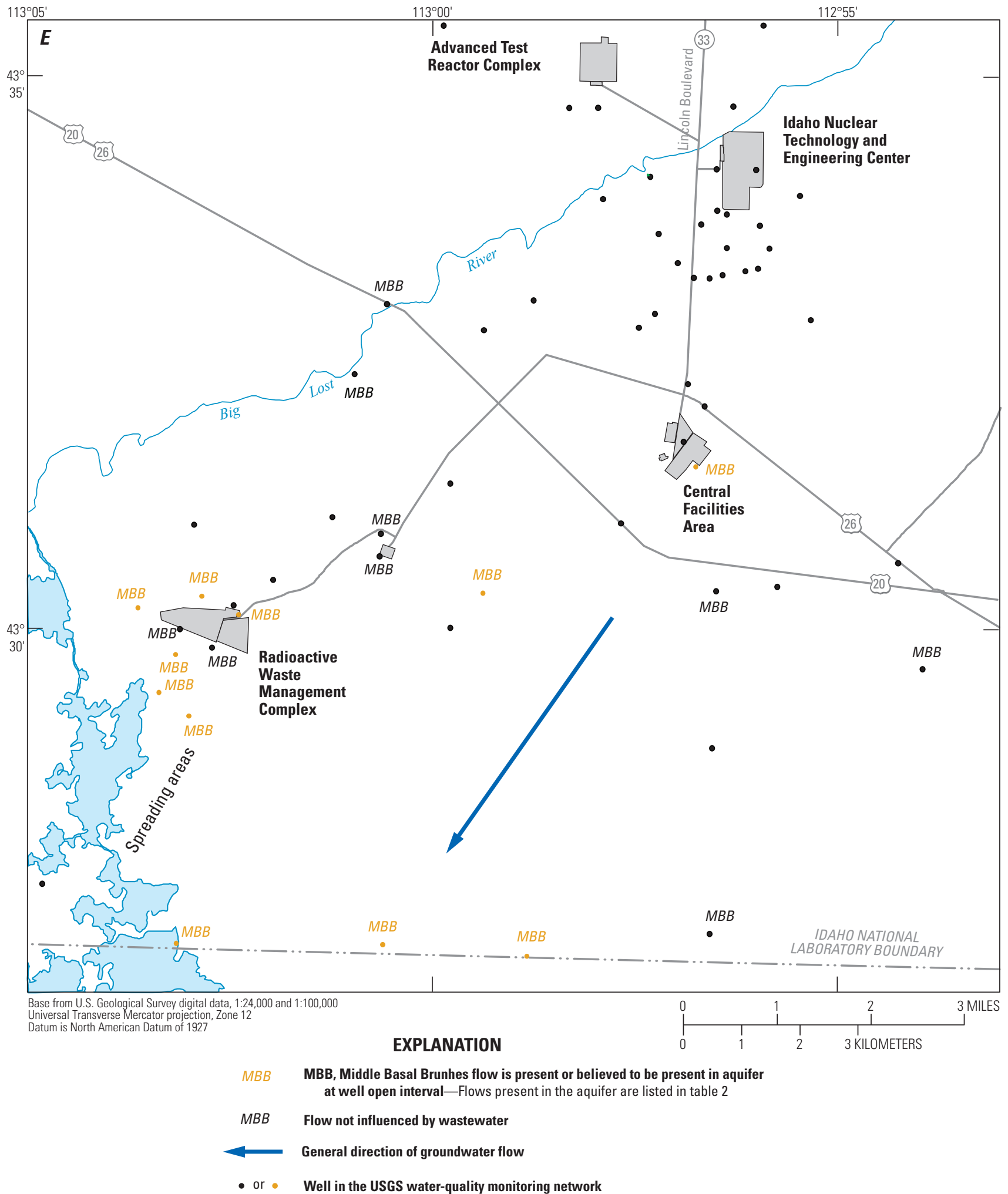

Figure 5.-Continued 


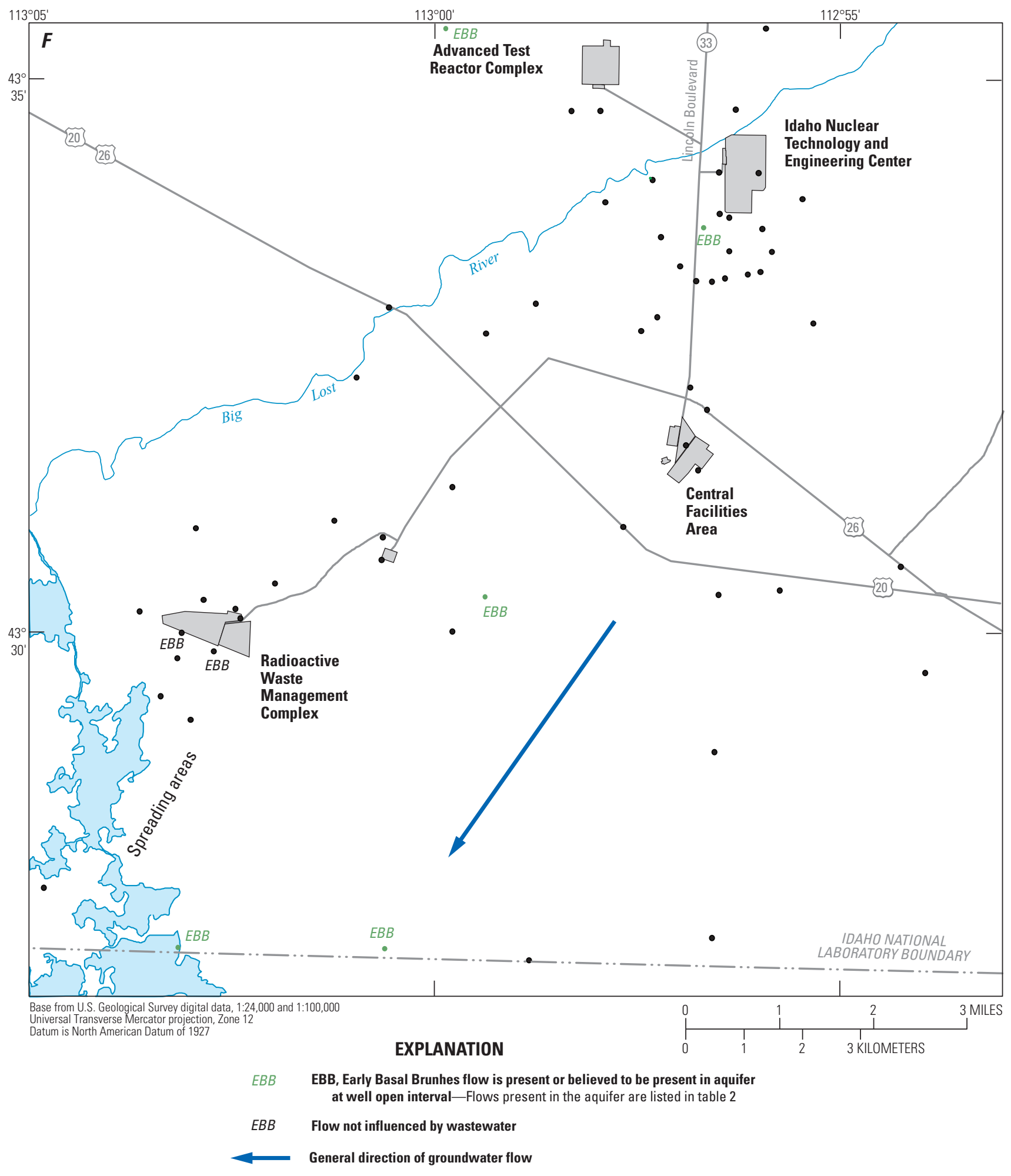

- or Well in the USGS water-quality monitoring network

Figure 5.-Continued 


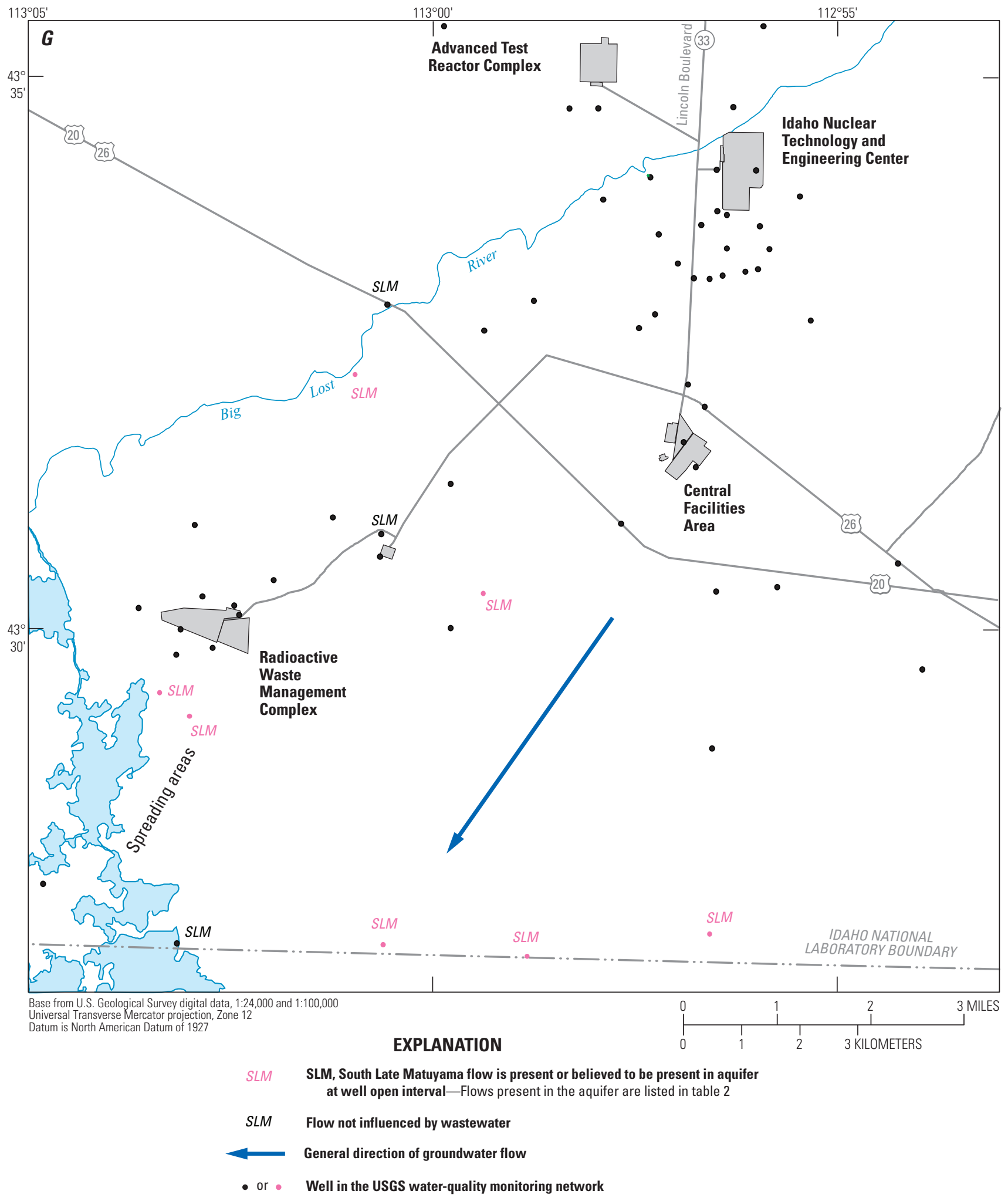

Figure 5.-Continued 


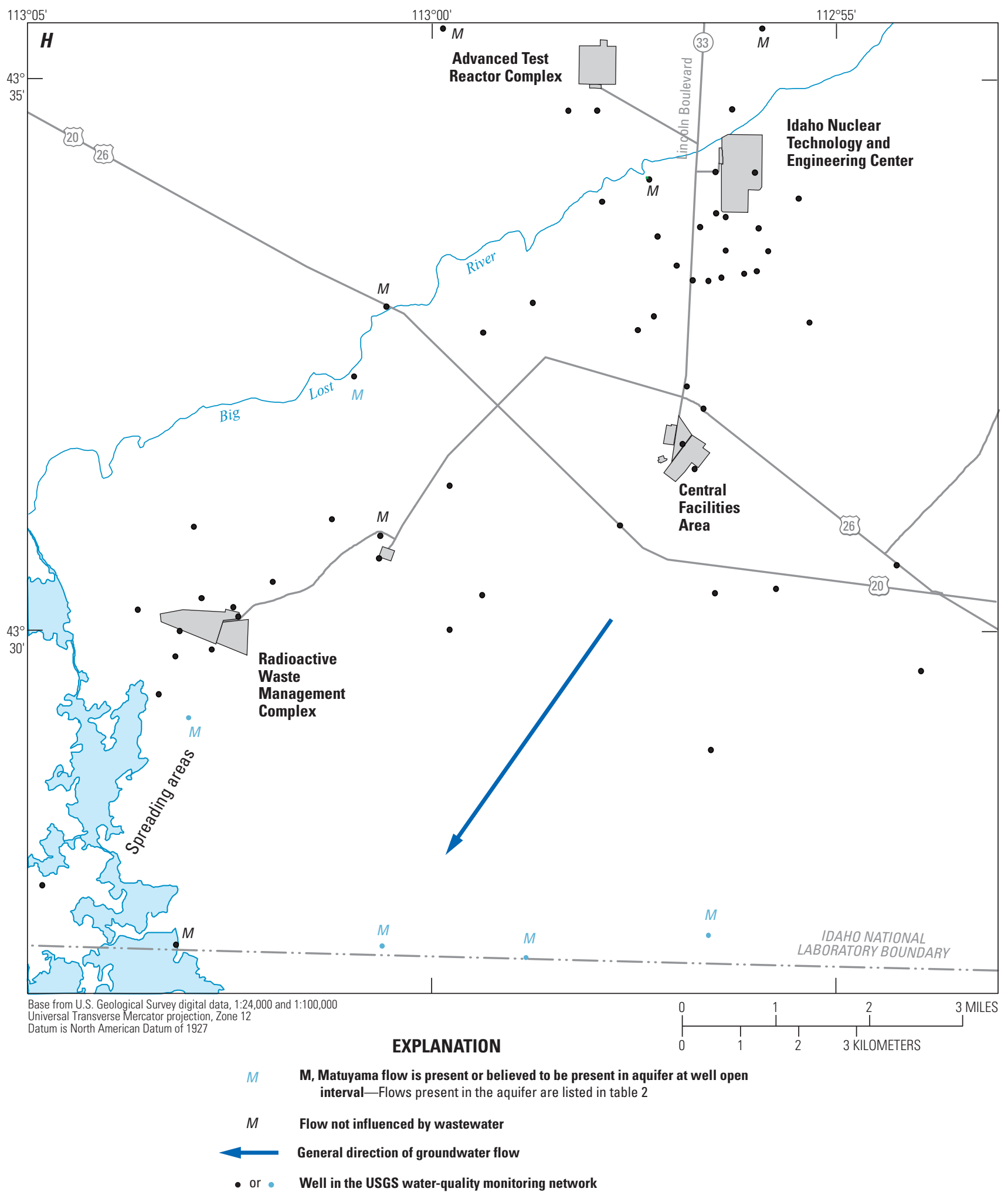

Figure 5.-Continued 


\section{Advanced Test Reactor Complex and Idaho Nuclear Technology and Engineering Center Area}

The basalt flows in the upper $150 \mathrm{ft}$ of the ESRP aquifer at INTEC (depth of the Idaho Chemical Processing Plant (ICPP) Disposal well where wastewater was discharged) consisted of the CFA Buried Vent flow and the AEC Butte flow. At the ATR Complex, where wastewater from long-term disposal in infiltration ponds would presumably move downward and intersect the top of the water table, the CFA Buried Vent flow probably occurs as the primary stratigraphic unit present; however, AEC Butte flow also could be present at some of the locations near the facility (Champion and others, 2011, pl. 1; Hodges and Champion, 2016, pl. 1).

Tritium concentrations greater than the lower estimated background concentration of $75 \mathrm{pCi} / \mathrm{L}$ (Orr and others, 1991) are assumed to indicate groundwater that has been influenced by wastewater disposal at the INL (fig. 4). Wells shown with tritium concentrations less than the laboratory detection level of $200 \mathrm{pCi} / \mathrm{L}$ have not been evaluated at a low enough detection level to determine whether wastewater constituents are influencing the water quality; however, evidence from concentrations of other chemical constituents such as chloride and volatile organic compounds mostly indicate that these wells probably are not affected by waste disposal. Almost all the wells at and near the ATR Complex and INTEC are influenced by wastewater disposal at the two facilities (this includes the wells north of highway 20/26 and north of CFA; fig. 4). An exception is ICPP Mon-A-166 (fig. 2), which was drilled as a downgradient monitoring well for new infiltration ponds southwest of INTEC, but does not show any influence from the ponds or from disposal from the ATR Complex, even though the Rifle Range well (fig. 2), farther downgradient and along the same flow path, does (Bartholomay and others, 2017). Both wells are presumed to have similar stratigraphy (CFA Buried Vent), but the Rifle Range Well is completed in more than $100 \mathrm{ft}$ of the ESRP aquifer, whereas ICPPMon-A-166 is completed in the upper $15 \mathrm{ft}$ of the aquifer (table 2).

Closer to where wastewater disposal occurred at INTEC and ATR Complex, almost all wells show wastewater influence in the upper part of the ESRP aquifer with wastewater present in both the CFA Buried Vent flow and AEC Butte flow (figs. 5B, 5C). Several wells near the ATR Complex and INTEC were not completed deep enough to sample more than the upper one or two basalt flows present in these areas. Well Middle 2050A is a multilevel monitoring well completed much deeper into the aquifer and located to the west of INTEC and southeast of the ATR Complex (fig. 1). Tritium data indicate minor wastewater influence in the areas of the aquifer that contain the CFA Buried Vent flow, the AEC Butte flow, and the Jaramillo flow (Bartholomay and others, 2015, fig. 19). Other data from well Middle 2050A indicate that the Late
Basal Brunhes, Early Basal Brunhes, and Matuyama flows are not influenced by wastewater (figs. 5D, 5F, 5E; Bartholomay and others, 2015, fig. 19).

\section{Central Facilities Area}

Historically, wastewater contamination at the CFA is attributed to disposal activities at the INTEC that has moved downgradient and affected the water quality of the aquifer near the CFA, although some of the nitrate present in CFA 2 and USGS 130 has been attributed to disposal from CFA to the old mercury ponds south of the CFA (Bartholomay and others, 2017, p. 54). Figure 4 shows several of the wells south and southeast of CFA (USGS 127, 83, 144, and Badging Facility) are not influenced by wastewater; however, wells farther south and southwest (USGS 104, 131A, and 106) are influenced by wastewater disposal.

Basalt stratigraphy indicates that the CFA Buried Vent flow is the predominant flow in the upper part of the ESRP aquifer at and south of CFA (fig. $5 B$ ). This unit seems to pinch out southeast of CFA (Champion and others, 2011, pl. 1). The younger Big Lost flow is present in all of the wells south, southeast, and southwest of CFA, and the older AEC Butte flow is present in the wells at and north of CFA (figs. 5A, 5B, $5 \mathrm{C}$, respectively).

The depth of open intervals in wells completed in the ESRP aquifer varies from about 100 to $250 \mathrm{ft}$ (table 2) in most wells, so wastewater detection is independent of the amount of open interval sampled. Most of the wells (USGS 127, 144, and Badging Facility well) that are not influenced by wastewater disposal do not have a known hydraulic conductivity (fig. 6), so it is not certain if hydraulic conductivity plays a role in the absence of wastewater constituents. However, because the CFA Buried Vent flow originated somewhere near CFA (Hodges and Champion, 2016), it is reasonable to consider the most massive part of the flow and thus least conductive part of the aquifer could occur just south of CFA, which may be why wells in that area do not show influence from wastewater disposal. Additionally, because of subsidence in the ESRP, the upper part of the aquifer in this area is completed in the Big Lost Flow unit, which is above the CFA Buried Vent flow where wastewater was presumably discharged at INTEC and the ATR Complex. It may be that wastewater influenced groundwater flow occurs in the deeper parts of the ESRP aquifer in wells south of CFA.

Multilevel monitoring well USGS 131A shows wastewater influence in all four zones sampled and the zones are completed in the CFA Buried Vent flow, the Early Basal Brunhes flow and the Jaramillo flows (Bartholomay and others, 2015, fig. 19). The Big Lost, Middle Basal Brunhes, South Late Matuyama, and Matuyama flows are also present in the aquifer in this well (table A21), but the zones in those flows were not sampled. This well is completed in about the upper $600 \mathrm{ft}$ of the aquifer and possibly is influenced by wastewater through its entire thickness. 


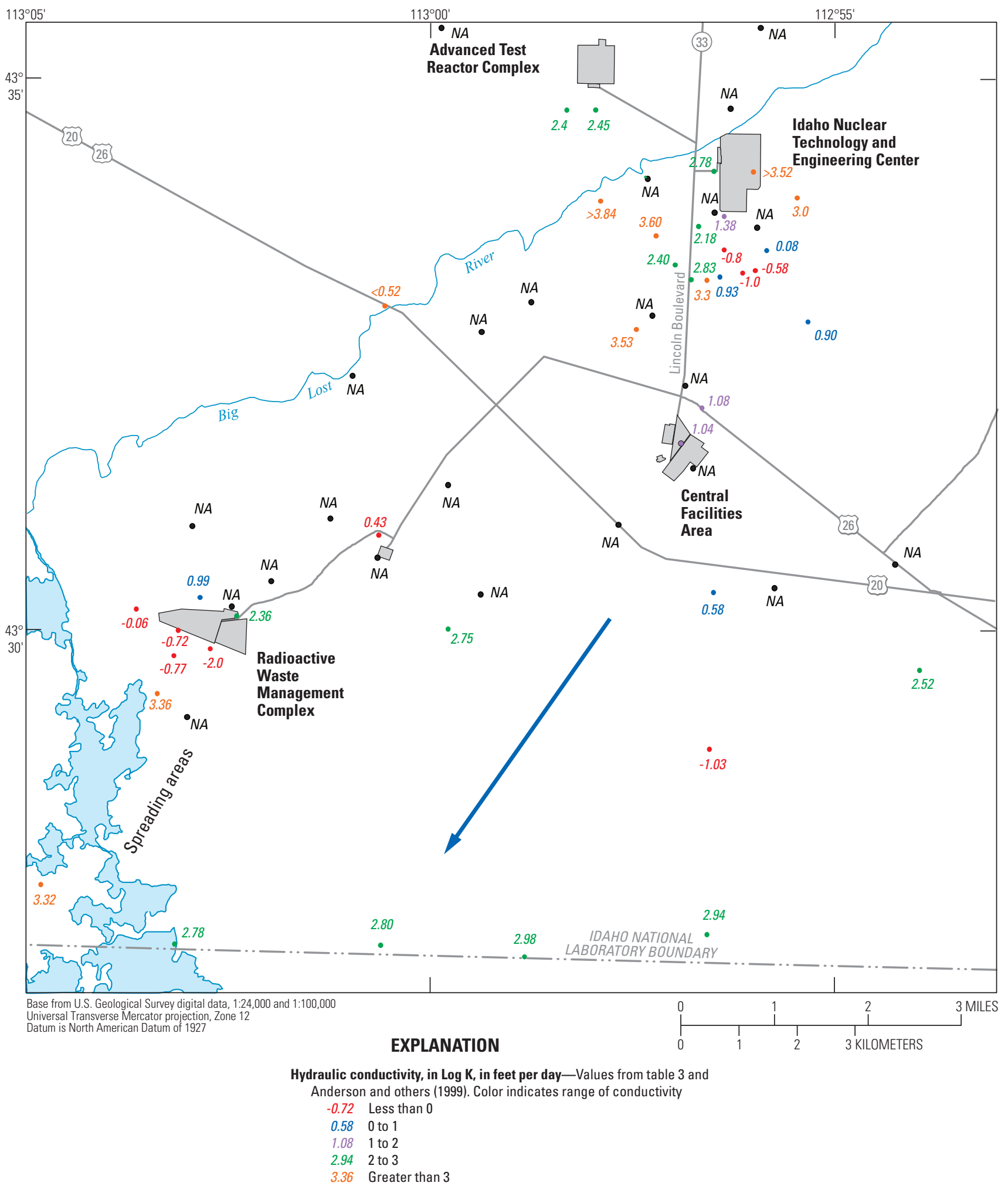

$\checkmark$ General direction of groundwater flow

- Well in the USGS water-quality monitoring network-Water samples analyzed for hydraulic conductivity. NA, not analyzed

Figure 6. Hydraulic conductivity measured in selected wells in the southwestern part of the Idaho National Laboratory, Idaho. 


\section{Radioactive Waste Management Complex Area}

At the RWMC, where water with contaminants from buried wastes would presumably move down through the unsaturated zone and pond on the surface of the water table, the CFA Buried Vent, Late Basal Brunhes, and Early Basal Brunhes all have been identified as the flow units at or near the water table in different cores (Champion and others, 2011, pl. 1; Hodges and Champion, 2016, pl. 1; appendix A). Additionally, wastewater disposal from the ATR Complex and possibly INTEC also have contributed to some of the wastewater contamination in monitoring wells around the RWMC (Davis and others, 2015; Bartholomay and others, 2017). Tritium was not detected in wells USGS 88, 89, 117, and 119 directly south of RWMC and EBR 1 and RWMC M13S northeast of RWMC (figs. 2 and 4), but concentrations were not analyzed at a low enough detection level to determine whether or not wastewater constituents are influencing the water quality. However, evidence from concentrations of other chemical constituents such as chloride and volatile organic compounds (VOCs) indicate that these wells probably are not affected by waste disposal. An exception is well USGS 88 (table 3) that has VOCs present in samples that indicate movement of water originating from buried waste at the RWMC (Bartholomay and others, 2017, table 8). Additionally, USGS 88 and 89 have chloride concentrations that are greater than background and the high chloride concentrations have been attributed to waste disposal at the INL (table 3; Gordon Rattray, U.S. Geological Survey, written commun., 2017). All four wells south of RWMC that do not show influence from tritium (USGS 88, 89, 117, and 119) all have low hydraulic conductivities (fig. 6), which may be limiting the movement of water into these wells.

Basalt stratigraphy indicates that the CFA Buried Vent flow is the predominant flow in the upper part of the ESRP aquifer at and near the RWMC because it is present in all the wells (fig. 5B). The Late Basal Brunhes flow is present in most wells north and northeast of RWMC (fig. 5D). The Middle Basal Brunhes flow is present in the ESRP aquifer in all but one of the wells near the RWMC and in two upgradient wells (fig. $5 E$ ). The Early Basal Brunhes flow is present in USGS 117 and 119 (fig. 5F). The South Late Matuyama flow is present in EBR 1, USGS 120 and 132, and the Matuyama flow is present in Middle 2051, EBR 1 and USGS 132 (fig. 5G, 5H; table 2).

Two multilevel monitoring wells completed in the upper 600-700 $\mathrm{ft}$ of the ESRP aquifer show wastewater-influenced water in several zones of the aquifer both northwest of RWMC (Middle 2051) and south of RWMC (USGS 132). Well Middle 2051 shows wastewater influence in the Matuyama and
Jaramillo flows (Bartholomay and others, 2015, fig. 19) and probably would have wastewater influence in the South Late Matuyama and Post Jaramillo flows if water samples were collected from those zones, given the nature of the stratigraphy in that well. The geology above a large sedimentary interbed in this well has the CFA Buried Vent flow, Late Basal Brunhes flow, and Middle Basal Brunhes flows that make up the upper part of the ESRP aquifer in this location. The water in this part of the aquifer is believed to be influenced by Big Lost River surface recharge (Bartholomay and Twining, 2010; Bartholomay and others, 2015) and no wastewater influence seems to be affecting the upper part of this well. USGS 132 shows wastewater influence in all 6 zones sampled; the zones are completed in the Middle and Early Basal Brunhes flows and the Matuyama flow (Bartholomay and others, 2015, fig. 19). The CFA Buried Vent flow is also present in the upper part of the aquifer in this well (table A22) and because wastewater is present in all the zones, wastewater is probably present in this flow unit.

\section{Southern Boundary of the Idaho National Laboratory}

Movement of buried wastes at the RWMC and wastewater disposal from the ATR Complex and INTEC have probably contributed to some of the wastewater contamination in the monitoring wells at the southern boundary of the INL (Bartholomay and Twining, 2010; Bartholomay and others, 2015; Davis and others, 2015). Basalt flows that show some wastewater influence along the southern boundary are in the G flow, Middle and Early Basal Brunhes flows, the South Late Matuyama flow and the Matuyama flow; however, the greatest influence appears to be in the South Late Matuyama flow (Bartholomay and others [2015, fig. 20]). Revised interpretation of the flows present in USGS 137A (Hodges and Champion, 2016) indicate the Big Lost flow, $\mathrm{G}$ flow, and the Early Basal Brunhes flows are influenced by wastewater, but not the South Late Matuyama as indicated by Bartholomay and others (2015). Wells USGS 137A, 105, 108 , and 103 all show wastewater influence in several zones; however, well USGS 135 (fig. 1) west of these wells does not show wastewater influence in any of the well zones in the over $400 \mathrm{ft}$ of ESRP aquifer in which it was completed. All four zones of USGS 135 were completed in the South Late Matuyama and Matuyama flows (table A25). The concentrations of wastewater constituents in deeper zones in wells USGS 105, 108, and 103 support the concept of groundwater flow deepening in the southwestern part of the INL as indicated by Ackerman and others (2010). 


\section{Summary and Conclusions}

Wastewater discharged to wells and ponds at the Advanced Test Reactor Complex (ATR Complex) and Idaho Nuclear Technology and Engineering Center (INTEC) and wastes buried in shallow pits and trenches at the Radioactive Waste Management Complex (RWMC) have contributed contaminants to the eastern Snake River Plain (ESRP) aquifer in the southwestern part of the Idaho National Laboratory (INL). This report investigated the correlation between subsurface stratigraphy in the southwestern part of the INL with information on the presence or absence of wastewater constituents to better understand how wastewater discharged at INL facilities are controlled by flow pathways in the aquifer Paleomagnetic inclination was used to identify subsurface basalt flows based on similar inclination measurements and polarity. Tritium concentrations were used to determine wastewater influence and were analyzed at two different laboratories using two different detection levels. For wells with tritium concentrations below detection levels, other chemical constituents also were examined to make a determination as to whether or not the well was influenced by wastewater disposal.

Drill cores were used to understand the basalt stratigraphy and some of the cored holes were completed as monitoring wells. For wells not cored, nearby core information was used to interpret local stratigraphy.

The basalt flows in the upper $150 \mathrm{ft}$ of the ESRP aquifer where wastewater was discharged at INTEC consisted of the CFA Buried Vent flow and the AEC Butte flow. At the ATR Complex, where wastewater would presumably pond on the surface of the water table, the CFA Buried Vent flow probably occurs as the primary stratigraphic unit present, although the AEC Butte flow also could be present at some of the locations. In wells closer to where wastewater disposal occurred at INTEC and ATR Complex, almost all the wells show wastewater influence in the upper part of the ESRP aquifer with wastewater present in the CFA Buried Vent flow and AEC Butte flow. The CFA Buried vent flow was present in the most wells analyzed in this study (52) and wastewater constituents were present in 83 percent of the wells. The AEC Butte flow was present in 14 of the wells analyzed and all 14 have wastewater influence. Several wells at and around the ATR Complex and INTEC were not completed deep enough to sample more than just the upper one or two flows (CFA Buried Vent and AEC Butte) present in these areas. The predominance of wastewater movement in these flow units is expected because they were the units that wastewater was discharged. A multilevel monitoring well completed in several basalt flows (Middle 2050A) has data that indicates minor wastewater influence in parts of the aquifer comprised of the CFA Buried Vent flow, the AEC Butte flow, and the Jaramillo flow.
Other data from this well shows that the Late Basal Brunhes, Early Basal Brunhes, and Matuyama flows are not influenced by wastewater.

Several of the wells south and southeast of CFA (USGS $127,83,144$, and the Badging Facility well) are not influenced by wastewater; however, wells farther south and southwest (USGS 104, 131A, and 106) are influenced by wastewater disposal. Basalt stratigraphy indicates that the CFA Buried Vent flow is the predominant flow in the upper part of the ESRP aquifer at and south of CFA. The younger Big Lost flow is present in all of the wells south, southeast, and southwest of CFA, and the AEC Butte flow is present in the wells at CFA and north of CFA. Most of the wells that are not influenced by wastewater disposal do not have a measured hydraulic conductivity, so whether hydraulic conductivity plays a role in the absence of wastewater constituents is not certain. However, given that the CFA Buried Vent flow originated somewhere around CFA, the most massive part of the flow and thus the least conductive part of the aquifer likely could occur just south of CFA. This may be why wells in that area do not show influence from wastewater disposal. Because of subsidence in the ESRP, the upper part of the aquifer in this area also is completed in the younger Big Lost Flow, which overlies the CFA buried Vent flow in which wastewater was presumably discharged at INTEC and the ATR Complex. It may be that wastewater influenced groundwater flow would occur in the deeper parts of the ESRP aquifer in wells south of CFA. A multilevel monitoring well USGS 131A completed in the upper 600 feet of the aquifer shows wastewater influence in all four zones sampled, and the zones are completed in the CFA Buried Vent flow along with the Early Basal Brunhes and the Jaramillo flows. The Big Lost, Middle Basal Brunhes, South Late Matuyama, and Matuyama flows also are present in the aquifer in this well, but no samples were collected from zones in those flows.

At the RWMC, where buried wastes presumably would move through the unsaturated zone and pond on the surface of the water table, CFA Buried Vent, Late Basal Brunhes, and Early Basal Brunhes all have been the basalt flow at or near the water table in different cores. All four wells south of RWMC that do not show influence from tritium (USGS 88, 89, 117, and 119) all have low hydraulic conductivities, which may be limiting the movement of water into these wells. Basalt stratigraphy indicates that the CFA Buried Vent flow is the predominant flow in the upper part of the ESRP aquifer at and near the RWMC because it is present in all the wells. The Late Basal Brunhes flow, Middle Basal Brunhes flow, Early Basal Brunhes flow, South Late Matuyama flow, and Matuyama flow also are present in various wells influenced by waste disposal. Two multilevel monitoring wells completed in the upper 600-700 ft of the ESRP aquifer show wastewater influenced water in several zones completed in the ESRP aquifer both northwest of RWMC (Middle 2051) and south of RWMC (USGS 132). 
Basalt flows that show some wastewater influence along the southern boundary are in the G flow, Middle and Early Basal Brunhes flows, the South Late Matuyama flow, and the Matuyama flow; however, the South Late Matuyama flow shows the most wastewater influence. Wells USGS 137A, 105,108 , and 103 all show wastewater influence in several basalt flows; however, USGS 135 west of these wells does not show any wastewater influence in any of the basalt flows in the more than $400 \mathrm{ft}$ of ESRP aquifer that it was completed. All four zones of USGS 135 were completed in the South Late Matuyama and Matuyama flows. The concentrations of wastewater constituents in deeper zones in wells USGS 105,108 , and 103 support the concept of groundwater flow deepening in the southwestern part of the INL.

\section{References Cited}

Ackerman, D.J., 1991, Transmissivity of the Snake River Plain aquifer at the Idaho National Engineering Laboratory, Idaho: U.S. Geological Survey Water-Resources Investigations Report 91-4058 (DOE/ID-22097), 35 p., accessed February 25, 2016, at https://pubs.er.usgs.gov/ publication/wri914058.

Ackerman, D.J., Rattray, G.W., Rousseau, J.P., Davis, L.C., and Orr, B.R., 2006, A conceptual model of ground-water flow in the eastern Snake River Plain aquifer at the Idaho National Laboratory and vicinity with implications for contaminant transport: U.S. Geological Survey Scientific Investigations Report 2006-5122 (DOE/ID-22198), 62 p., accessed May 15, 2017 at https://pubs.er.usgs.gov/usgspubs/ sir/sir20065122.

Ackerman, D.J., Rousseau, J.P., Rattray, G.W., and Fisher, J.C., 2010, Steady-state and transient models of groundwater flow and advective transport, Eastern Snake River Plain aquifer, Idaho National Laboratory and vicinity, Idaho: U.S. Geological Survey Scientific Investigations Report 2010-5123, (DOE/ID-22209), 220 p., accessed February 25, 2016, at https://pubs.usgs.gov/sir/2010/5123/.

Anders, M.H., and Sleep, N.H., 1992, Magmatism and extension-The terminal and mechanical effects of the Yellowstone hotspot: Journal of Geophysical Research, v. 97 , no. B11, p. 15,379-15,393.

Anderson, S.R., 1991, Stratigraphy of the unsaturated zone and uppermost part of the Snake River Plain aquifer at the Idaho Chemical Processing Plant and Test Reactor Area, Idaho National Engineering Laboratory, Idaho: U.S. Geological Survey Water-Resources Investigations Report 91-4010 (DOE/ID 22095), 71 p., accessed February 25, 2016, at https://pubs.er.usgs.gov/publication/wri914010.
Anderson, S.R., Ackerman, D.J., Liszewski, M.J., and Freiburger, R.M., 1996, Stratigraphic data for wells at and near the Idaho National Engineering Laboratory, Idaho: U.S. Geological Survey Open-File Report 96-248 (DOE/ID22127), 27 p. and 1 diskette, accessed February 25, 2016, at https://pubs.er.usgs.gov/publication/ofr96248.

Anderson, S.R., and Bartholomay, R.C., 1995, Use of natural gamma logs and cores for determining the stratigraphic relations of basalt and sediment at the Radioactive Waste Management Complex, Idaho National Engineering Laboratory, Idaho: Journal of the Idaho Academy of Science, v. 31, issue 1, p. 1-10.

Anderson, S.R., and Bowers, Beverly, 1995, Stratigraphy of the unsaturated zone and uppermost part of the Snake River Plain aquifer at Test Area North, Idaho National Engineering Laboratory, Idaho: U.S. Geological Survey Water-Resources Investigations Report 95-4130 (DOE/ ID-22122), $47 \mathrm{p}$.

Anderson, S.R., Kuntz, M.A., and Davis, L.C., 1999, Geologic controls of hydraulic conductivity in the Snake River Plain aquifer at and near the Idaho National Engineering and Environmental Laboratory, Idaho: U.S. Geological Survey Water-Resources Investigations Report 99-4033 (DOE/ ID-22155), 38 p., accessed February 25, 2016, at https:// pubs.er.usgs.gov/publication/wri994033.

Anderson, S.R., and Lewis, B.D., 1989, Stratigraphy of the unsaturated zone at the Radioactive Waste Management Complex, Idaho National Engineering Laboratory, Idaho: U.S. Geological Survey Water-Resources Investigations Report 89-4065 (DOE/ID-22080), 54 p., accessed February 25, 2016, at https://pubs.er.usgs.gov/publication/ wri894065.

Anderson, S.R., and Liszewski, M.J., 1997, Stratigraphy of the unsaturated zone and the Snake River Plain aquifer at and near the Idaho National Engineering Laboratory, Idaho: U.S. Geological Survey Water-Resources Investigations Report 97-4183 (DOE/ID-22142), 65 p., accessed February 25, 2016, at https://pubs.er.usgs.gov/publication/ wri974183.

Anderson, S.R., Liszewski, M.J., and Ackerman, D.J., 1996, Thickness of surficial sediment at and near the Idaho National Engineering Laboratory, Idaho: U.S. Geological Survey Open-File Report 96-330 (DOE/ID-22128), 16 p.

Bartholomay, R.C., Davis, L.C., Fisher, J.C., Tucker, B.J., and Raben, F.A., 2012, Water-quality characteristics and trends for selected sites at and near the Idaho National Laboratory, Idaho, 1949-2009: U.S. Geological Survey Scientific Investigations Report 2012-5169 (DOE/ID 22219), 68 p. [Also available at https://pubs.usgs.gov/sir/2012/5169/.] 
Bartholomay, R.C., and Hall, L.F., 2016, Evaluation of background concentrations of selected chemical and radiochemical constituents in water from the eastern Snake River Plain aquifer at and near the Idaho National Laboratory, Idaho: U.S. Geological Survey Scientific Investigations Report 2016-5056 (DOE/ID-22237), 19 p., https://doi.org/10.3133/sir20165056.

Bartholomay, R.C., Hopkins, C.B., and Maimer, N.V., 2015, Chemical constituents in groundwater from multiple zones in the eastern Snake River Plain aquifer, Idaho National Laboratory, Idaho, 2009-13: U.S. Geological Survey Scientific Investigations Report 2015-5002 (DOE/ ID-22232), 110 p., accessed February 25, 2016, at https:// pubs.er.usgs.gov/publication/sir20155002.

Bartholomay, R.C., Maimer, N.V., Rattray, G.W., and Fisher, J.C., 2017, An update of hydrologic conditions and distribution of selected constituents in water, eastern Snake River Plain aquifer and perched groundwater zones, Idaho National Laboratory, Idaho, emphasis 2012-15: U.S. Geological Survey Scientific Investigations Report 2017-5021 (DOE/ID-22242), 87 p., https://doi.org/10.3133/ sir20175021.

Bartholomay, R.C., Maimer, N.V., and Wehnke, A.J., 2014, Field methods and quality-assurance plan for water-quality activities and water level measurements, U.S. Geological Survey, Idaho National Laboratory, Idaho: U.S. Geological Survey Open-File Report 2014-1146 (DOE/ID-22230), 66 p., https://pubs.usgs.gov/of/2014/1146/.

Bartholomay, R.C., Tucker, B.J., Ackerman, D.J., and Liszewski, M.J., 1997, Hydrologic conditions and distribution of selected radiochemical and chemical constituents in water, Snake River Plain aquifer, Idaho National Engineering Laboratory, Idaho, 1992 through 1995: U.S. Geological Survey Water-Resources Investigations Report 97-4086 (DOE/ID-22137), 57 p., https://pubs.er.usgs.gov/publication/wri974086.

Bartholomay, R.C., and Twining, B.V., 2010, Chemical constituents in groundwater from multiple zones in the eastern Snake River Plain aquifer at the Idaho National Laboratory, Idaho: U.S. Geological Survey Scientific Investigations Report 2010-5116 (DOE/ID 22211), 81 p. [Also available at https://pubs.er.usgs.gov/publication/ sir20105116.]

Bestland, E.A., Link, P.K., Lanphere, M.A., and Champion, D.E., 2002, Paleoenvironments of sedimentary interbeds in the Pliocene and Quaternary Big Lost Trough (eastern Snake River Plain, Idaho), in Link, P.K., and Mink, L.L., eds., Geology, hydrogeology and environmental remediation, Idaho National Engineering and Environmental Laboratory, Eastern Snake River Plain, Idaho: Boulder, Colo., Geological Society of America Special Paper 353, p. 27-44.
Blair, J.J., 2002, Sedimentology and stratigraphy of sediments of the Big Lost trough subsurface from selected coreholes at the Idaho National Engineering and Environmental Laboratory: Pocatello, Idaho, Idaho State University, Master's thesis, $148 \mathrm{p}$.

Braile, L.W., Smith, R.B., Ansorge, J., Baker, M.R., Sparlin, M.A., Prodehl, C., Schilly, M.M., Healy, J.H., Mueller, S., and Olsen, K.H., 1982, The Yellowstone-Snake River Plain seismic profiling experiment-Crustal structure of the Eastern Snake River Plain: Journal of Geophysical Research, v. 87, no. B4, p. 2,597-2,609.

Busenberg, Eurybiades, Plummer, L.N., and Bartholomay, R.C., 2001, Estimated age and source of the young fraction of ground water at the Idaho National Engineering and Environmental Laboratory: U.S. Geological Survey Water-Resources Investigations Report 2001-4265 (DOE/ID-22177), 144 p., https://pubs.er.usgs. gov/publication/wri014265.

Cecil, L.D., Welhan, J.A., Green, J.R., Frape, S.K., and Sudicky, E.R., 2000, Use of chlorine-36 to determine regional-scale aquifer dispersivity, eastern Snake River Plain aquifer, Idaho/USA: Nuclear Instruments and Methods in Physics Research, section B, v. 172, issues 1-4, p. 679-687.

Champion, D.E., Dalrymple, G.B., and Kuntz, M.A., 1981, Radiometric and paleomagnetic evidence for the Emperor reversed polarity event at $0.46 \pm 0.05 \mathrm{~m}$.y. in basalt lava flows from the eastern Snake River Plain, Idaho: Geophysical Research Letters, v. 8, no. 10, p. 1,055-1,058.

Champion, D.E., Davis, L.C., Hodges, M.K.V., and Lanphere, M.A., 2013, Paleomagnetic correlation and ages of basalt flow groups in coreholes at and near the Naval Reactors Facility, Idaho National Laboratory, Idaho: U.S. Geological Survey Scientific Investigations Report 2013-5012 (DOE/ ID-22223), 48 p.

Champion, D.E., and Greeley, R., 1978, Geology of the Wapi Lava Field, Idaho, in Greeley, R., and King, J.S., eds., Volcanism of the Snake River Plain, a comparative planetary geology guidebook: Washington, D.C., Office of Planetary Geology, National Aeronautics and Space Administration, p. 133-152.

Champion, D.E., and Herman, T.C., 2003, Paleomagnetism of basaltic lava flows in coreholes ICPP-213, ICPP-214, ICPP215, and USGS 128 near the vadose zone research park, Idaho Nuclear Technology and Engineering Center, Idaho National Engineering and Environmental Laboratory, Idaho: U.S. Geological Survey Open-File Report 2003-483 (DOE/ ID-22189), $21 \mathrm{p}$. 
Champion, D.E., Hodges, M.K.V., Davis, L.C., and Lanphere, M.A., 2011, Paleomagnetic correlation of surface and subsurface basaltic lava flows and flow groups in the southern part of the Idaho National Laboratory, Idaho, with paleomagnetic data tables for drill cores: U.S. Geological Survey Scientific Investigations Report 2011-5049, 34 p., 1 pl. (DOE/ID-22214), accessed February 25, 2016, at https://pubs.er.usgs.gov/publication/sir20115049.

Champion, D.E., Lanphere, M.A., Anderson, S.R., and Kuntz, M.A., 2002, Accumulation and subsidence of late Pleistocene basaltic lava flows of the eastern Snake River Plain, Idaho, in Link, P.K., and Mink, L.L., eds., Geology, hydrogeology, and environmental remediation-Idaho National Engineering and Environmental Laboratory, Eastern Snake River Plain, Idaho: Boulder, Colo., Geological Society of America Special Paper 353, p. 175192.

Champion, D.E., Lanphere, M.A., and Kuntz, M.A., 1988, Evidence for a new geomagnetic reversal from lava flows in Idaho-Discussion of short polarity reversals in the Brunhes and late Matuyama polarity chrons: Journal of Geophysical Research, v. 93, no. B10, p. 11,667-11,680.

Davis, L.C., Bartholomay, R.C., Fisher, J.C., and Maimer, N.V., 2015, Water-quality characteristics and trends for selected wells possibly influenced by wastewater disposal at the Idaho National Laboratory, Idaho, 1981-2012: U.S. Geological Survey Scientific Investigations Report 2015-5003 (DOE/ID-22233), 106 p.

Davis, L.C., Hannula, S.R., and Bowers, Beverly, 1997, Procedures for use of, and drill cores and cuttings available for study at, the Lithologic Core Storage Library, Idaho National Engineering Laboratory, Idaho: U.S. Geological Survey Open-File Report 97-124 (DOE/ID-22135), 31 p., accessed February 25, 2016, at https://pubs.er.usgs.gov/ publication/ofr97124.

Duke, C.L., Roback, R.C., Reimus, P.W., Bowman, R.S., McLing, T.L., Baker, K.E., and Hull, L.C., 2007, Elucidation of flow and transport processes in a variably saturated system of interlayered sediment and fractured rock using tracer tests: Vadose Zone Journal, v. 6, no. 4, p. $855-867$.

Garabedian, S.P., 1986, Application of a parameter-estimation technique to modeling the regional aquifer underlying the eastern Snake River Plain, Idaho: U.S. Geological Survey Water Supply Paper 2278, 60 p., https://pubs.er.usgs.gov/ publication/wsp2278.
Geslin, J.K., Link, P.K., Riesterer, J.W., Kuntz, M.A., and Fanning, C.M., 2002, Pliocene and Quaternary stratigraphic architecture and drainage systems of the Big Lost Trough, northeastern Snake River Plain, Idaho, in Link, P.K., and Mink, L.L., eds., Geology, hydrogeology, and environmental remediation-Idaho National Engineering and Environmental Laboratory, Eastern Snake River Plain, Idaho: Boulder, Colo., Geological Society of America Special Paper 353, p. 11-26.

Greeley, Ronald, 1982, The style of basaltic volcanism in the eastern Snake River Plain, Idaho, in Bonnichsen, Bill, and Breckinridge, R.M., eds., Cenozoic geology of Idaho: Idaho Bureau of Mines and Geology Bulletin 26, p. 407-421.

Grimm-Chadwick, Claire, 2004, Petrogenesis of an evolved olivine tholeiite and chemical stratigraphy of cores USGS 127, 128, and 129, Idaho National Engineering and Environmental Laboratory: Pocatello, Idaho, Idaho State University, Master's thesis, 100 p., plus apps.

Hackett, W.R., and Smith, R.P., 1992, Quaternary volcanism, tectonics, and sedimentation in the Idaho National Engineering Laboratory area, in Wilson, J.R., ed., Field guide to geologic excursions in Utah and adjacent areas of Nevada, Idaho, and Wyoming: Utah Geological Survey Miscellaneous Publication 92-3, p. 1-18.

Hagstrum, J.T., and Champion, D.E., 2002, A Holocene paleosecular variation record from ${ }^{14} \mathrm{C}$-dated volcanic rocks in western North America: Journal of Geophysical Research, v. 107, no. B1, 2,025, 14 p., doi:10.1029/2001JB000524.

Hodges, M.K.V., and Champion, D.E., 2016, Paleomagnetic correlation of basalt flows in selected coreholes near the Advanced Test Reactor Complex, the Idaho Nuclear Technology and Engineering Center, and along the southern boundary, Idaho National Laboratory, Idaho: U.S. Geological Survey Scientific Investigations Report 2016-5131 (DOE/ID-22240), 65 p., 1 pl.

Hodges, M.K.V., Orr, S.M., Potter, K.E., and LeMaitre, Tynan, 2012, Construction diagrams, geophysical logs, and lithologic descriptions for boreholes USGS 103, 105, 108, 131, 135, NRF-15, and NRF-16, Idaho National Laboratory, Idaho: U.S. Geological Survey Data Series 660, 34 p., accessed February 25, 2016, at https://pubs.er.usgs.gov/ publication/ds660.

Hodges, M.K.V., Turrin, B.D., Champion, D.E., and Swisher, C.C., III, 2015, New argon-argon $\left({ }^{40} \mathrm{Ar} /{ }^{39} \mathrm{Ar}\right)$ radiometric age dates from selected subsurface basalt flows at the Idaho National Laboratory, Idaho: U.S. Geological Survey Scientific Investigations Report 2015-5028 (DOE/ID 22234), 25 p., accessed February 25, 2016, at https://pubs. er.usgs.gov/publication/sir20155028. 
Hughes, S.S., Smith, R.P., Hackett, W.R., and Anderson, S.R., 1999, Mafic volcanism and environmental geology of the eastern Snake River Plain in Hughes, S.S., and Thackray, G.D., eds., Guidebook to the geology of eastern Idaho: Pocatello, Idaho, Idaho Museum of Natural History, p. $143-168$.

Kellogg, K.S., Harlan, S.S., Mehnert, H.H., Snee, L.W., Pierce, K.S., Hackett, W.R., and Rodgers, D.W., 1994, Major 10.2-Ma rhyolitic volcanism in the eastern Snake River Plain, Idaho-Isotopic age and stratigraphic setting of the Arbon Valley Tuff Member of the Starlight Formation: U.S. Geological Survey Bulletin 2091, 18 p., accessed February 25, 2016, at https://pubs.er.usgs.gov/publication/ b2091.

Kirschvink, J.L., 1980, The least-squares line and plane and the analysis of palaeomagnetic data: Geophysical Journal International, v. 62, p. 699-718.

Kuntz, M.A., 1978, Geology of the Big Southern Butte area, eastern Snake River Plain, and potential volcanic hazards to the Radioactive Waste Management Complex, and other waste storage and reactor facilities at the Idaho National Engineering Laboratory, Idaho, with a section on Statistical treatment of the age of lava flows, by John O. Kork: U.S. Geological Survey Open-File Report 78-691, 70 p.

Kuntz, M.A., 1992, A model-based perspective of basaltic volcanism, eastern Snake River Plain, Idaho in Link, P.K., Kuntz, M.A., and Platt, L.B., Regional geology of eastern Idaho and western Wyoming: Geological Society of America Memoir 179, p. 289-304.

Kuntz, M.A., Covington, H.R., and Schorr, L.J., 1992, An overview of basaltic volcanism of the eastern Snake River Plain, Idaho, in Link, P.K., Kuntz, M.A., and Platt, L.B., Regional geology of eastern Idaho and western Wyoming: Geological Society of America Memoir 179, p. 227-267.

Kuntz, M.A., Dalrymple, G.B., Champion, D.E., and Doherty, D.J., 1980, An evaluation of potential volcanic hazards at the Radioactive Waste Management Complex, Idaho National Engineering Laboratory, Idaho: U.S. Geological Survey Open-File Report 80-388, 63 p., 1 map, accessed February 25, 2016, at https://pubs.er.usgs.gov/ publication/ofr80388.

Kuntz, M.A., Link, P.K., Boyack, D.L., Geslin, J.K., Mark, L.E., Hodges, M.K.V., Kauffman, M.E., Champion, D.E., Lanphere, M.R., Rodgers, D.W., and Anders, M.H., 2003, Geologic map of the northern and central parts of the Idaho National Engineering and Environmental Laboratory, Eastern Idaho: Idaho Geological Survey Publication GM-35, color map with 14-p. booklet, scale 1:50,000, accessed March 3, 2016, at https:// www.idahogeology.org/Products/MapCatalog/default. asp?switch $=$ title \&value $=$ GM-35.
Kuntz, M.A., Skipp, Betty, Champion, D.E., Gans, P.B., Van Sistine, D.P., and Snyders, S.R., 2007, Geologic map of the Craters of the Moon 30' x 60' quadrangle, Idaho: U.S. Geological Survey Scientific Investigations Map 2969, 64-p. pamphlet, 1 plate, scale 1:100,000. [Also available at https://pubs.usgs.gov/sim/2007/2969/.]

Kuntz, M.A., Skipp, Betty, Lanphere, M.A., Scott, W.E., Pierce, K.L., Dalrymple, G.B., Champion, D.E., Embree, G.F., Page, W.R., Morgan, L.A., Smith, R.P., Hackett, W.R., and Rodgers, D.W., 1994, Geologic map of the Idaho National Engineering Laboratory and adjoining area, eastern Idaho: U.S. Geological Survey Miscellaneous Investigations Series I-2330, scale 1:100,000.

Kuntz, M.A., Spiker, E.C., Rubin, Meyer, Champion, D.E., and Lefebvre, R.H., 1986, Radiocarbon studies of latest Pleistocene and Holocene lava flows of the Snake River Plain, Idaho-Data, lessons, interpretations: Quaternary Research, v. 25, no. 2, p. 163-176.

Lanphere, M.A., Champion, D.E., and Kuntz, M.A., 1993, Petrography, age, and paleomagnetism of basalt lava flows in coreholes Well 80, NRF 89-04, NRF 89-05, and ICPP 123, Idaho National Engineering Laboratory: U.S. Geological Survey Open-File Report 93-0327, 40 p.

Lanphere, M.A., Kuntz, M.A., and Champion, D.E., 1994, Petrography, age, and paleomagnetism of basaltic lava flows in coreholes at Test Area North (TAN), Idaho National Engineering Laboratory: U.S. Geological Survey Open-File Report 94-686, 49 p.

Mann, L.J., 1986, Hydraulic properties of rock units and chemical quality of water for INEL-1-A 10,365-foot deep test hole drilled at the Idaho National Engineering Laboratory, Idaho: U.S. Geological Survey Water-Resources Investigations Report 86-4020 (IDO-22070), 23 p., https:// pubs.er.usgs.gov/publication/wri864020.

Mann, L.J., and Beasley, T.M., 1994, Iodine-129 in the Snake River Plain aquifer at and near the Idaho National Engineering Laboratory, Idaho, 1990-91: U.S. Geological Survey Water-Resources Investigations Report 94-4053 (DOE/ID-22115), 27 p.

Mazurek, John, 2004, Genetic controls on basalt alteration within the eastern Snake River Plain aquifer system, Idaho: Pocatello, Idaho, Idaho State University, Master's thesis, 132 p. and apps.

McCurry, M.O., and Hughes, S.S., 2006, Rhyolite volcanic fields of the Yellowstone-Snake River Plain hot spot trackDoes the Picabo Field exist?: Eos, Transactions, American Geophysical Union, v. 87, no. 52, Suppl. 26, Abstract V51D-1705. 
McQuarrie, Nadine, and Rodgers, D.W., 1998, Subsidence of a volcanic basin by flexure and lower crustal flow-The eastern Snake River Plain, Idaho: Tectonics, v. 17, p. 203220.

Miller, M.L., 2007, Basalt stratigraphy of corehole USGS-132 with correlations and petrogenetic interpretations of the B Flow Group, Idaho National Laboratory, Idaho: Pocatello, Idaho, Idaho State University Master's thesis, 69 p., 1 app., and $1 \mathrm{pl}$.

Morgan, L.A., and McIntosh, W.C., 2005, Timing and development of the Heise volcanic field, Snake River Plain, Idaho, western USA: Geological Society of America Bulletin, v. 117, no. 3 of 4, p. 288-306.

Morse, L.H., and McCurry, M.O., 2002, Genesis of alteration of Quaternary basalts within a portion of the eastern Snake River Plain aquifer, in Link, P.K., and Mink, L.L., eds., Geology, hydrogeology, and environmental remediationIdaho National Engineering and Environmental Laboratory, eastern Snake River Plain, Idaho: Boulder, Colo., Geological Society of America Special Paper 353, p. 213-224.

Nimmo, J.R., Perkins, K.S., Rose, P.A., Rousseau, J.P., Orr, B.R., Twining, B.V., and Anderson, S.R., 2002, Kilometer-scale rapid transport of naphthalene sulfonate tracer in the unsaturated zone at the Idaho National Engineering and Environmental Laboratory: Vadose Zone Journal, v. 1, issue 1, p. 89-101.

Ogg, J.G., and Smith, A.G., 2004, The geomagnetic polarity time scale, in Gradstein, F.M., Ogg, J.G., and Smith, A.G., eds., 2004, A geologic time scale 2004: New York, Cambridge University Press, 589 p.

Orr, B.R., Cecil, L.D., and Knobel, L.L., 1991, Background concentrations of selected radionuclides, organic compounds, and chemical constituents in ground water in the vicinity of the Idaho National Engineering Laboratory: U.S. Geological Survey Water-Resources Investigations Report 91-4015 (DOE/ID-22094), 52 p., https://pubs. er.usgs.gov/publication/wri914015.

Peng, Xiaohua, and Humphreys, E.D., 1998, Crustal velocity structure across the eastern Snake River Plain and the Yellowstone Swell: Journal of Geophysical Research, v. 103 , no. B4, p. 7,171-7,186.

Pierce, K.L., and Morgan, L.A., 1992, The track of the Yellowstone hot spot, in Link, P.K., Kuntz, M.A., and Platt, L.B., eds., Regional geology of eastern Idaho and western Wyoming: Geological Society of America Memoir 179 , p. $1-53$.
Pierce, K.L., Morgan, L.A., and Saltus, R.W., 2002, Yellowstone plume head-Postulated tectonic relations to the Vancouver Slab, continental boundaries, and climate, in Bonnichsen, Bill, White, C.M., and McCurry, M.O., eds., Tectonic and magmatic evolution of the Snake River Plain volcanic province: Idaho Geological Survey Bulletin 30, p. 5-29.

Plummer, L.N., Rupert, M.G., Busenberg, Eurybiades, and Schlosser, P., 2000, Age of irrigation water in ground water from the eastern Snake River Plain aquifer, South-central Idaho: Ground Water, v. 38, no. 2, p. 264-283.

Reed, M.F., Bartholomay, R.C., and Hughes, S.S., 1997, Geochemistry and stratigraphic correlation of basalt lavas beneath the Idaho Chemical Processing Plant, Idaho National Engineering Laboratory: Environmental Geology, v. 30, nos. 1-2, p. 108-118.

Rightmire, C.T., and Lewis, B.D., 1987, Geologic data collected and analytical procedures used during a geochemical investigation of the unsaturated zone, Radioactive Waste Management Complex, Idaho National Engineering Laboratory, Idaho: U.S. Geological Survey Open-File Report 87-0246 (DOE/ID-22072), 83 p.

Robertson, J.B., Schoen, Robert, and Barraclough, J.T., 1974, The influence of liquid waste disposal on the geochemistry of water at the National Reactor Testing Station, Idaho, 1952-1970: U.S. Geological Survey Open-File Report 73-238 (IDO-22053), $231 \mathrm{p}$.

Rodgers, D.W., Ore, H.T., Bobo, R.T., McQuarrie, Nadine, and Zentner, Nick, 2002, Extension and subsidence of the eastern Snake River Plain, Idaho, in Bonnichsen, Bill, White, C.M., and McCurry, Michael, eds., Tectonic and magmatic evolution of the Snake River Plain Volcanic Province: Idaho Geological Survey Bulletin 30, p. 121-155.

Russell, I.C., 1902, Geology and water resources of the Snake River plains of Idaho: U.S. Geological Survey Series Bulletin Report Number 199, 192 p.

Scarberry, K.C., 2003, Volcanology, geochemistry, and stratigraphy of the F Basalt Flow Group, eastern Snake River Plain, Idaho: Pocatello, Idaho, Idaho State University, Master's thesis, 139 p., 1 pl.

Shervais, J.W., Vetter, S.K., and Hanan, B.B., 2006, A layered mafic sill complex beneath the eastern Snake River PlainEvidence from cyclic geochemical variations in basalt: Geology, v. 34, no. 5, p. 365-368. 
Skipp, Betty, Snider, L.G., Janecke, S.U., and Kuntz, M.A., 2009, Geologic map of the Arco $30 \times 60$ minute quadrangle, south-central Idaho: Idaho Geological Survey Geologic Map GM-47, map scale 1:100,000 and 42 p. booklet, accessed March 3, 2016, at https:/www.idahogeology.org/Products/ MapCatalog/default.asp?switch=title \&value=GM-47.

Stroup, C.N., Welhan, J.A., and Davis, L.C., 2008, Statistical stationarity of sediment interbed thicknesses in a basalt aquifer, Idaho National Laboratory, eastern Snake River Plain, Idaho: U.S. Geological Survey Scientific Investigations Report 2008-5167 (DOE/ID-22204), 20 p.

Tauxe, Lisa, Luskin, Casey, Selkin, Peter, Gans, Phillip, and Calvert, Andy, 2004, Paleomagnetic results from the Snake River Plain - Contribution to the time-and field global database: $\mathrm{G}^{3}$ - Geochemistry, Geophysics, and Geosystems, v. 5, no. 8, 19 p., doi:10.1029/2003GC000661.

Twining, B.V., Hodges, M.K.V., and Orr, Stephanie, 2008, Construction diagrams, geophysical logs, and lithologic descriptions for boreholes USGS 126a, 126b, 127, 128, 129, 130, 131, 132, 133, and 134, Idaho National Laboratory, Idaho: U.S. Geological Survey Data Series Report 350 (DOE/ID-22205), 27 p., and apps., accessed February 25, 2016, at https://pubs.usgs.gov/ds/350/.

U.S. Geological Survey, 1985, National water summary, 1984-Hydrologic events, selected water-quality trends, and ground-water resources: U.S. Geological Survey WaterSupply Paper 2275, 467 p. [Also available at https://pubs. er.usgs.gov/publication/wsp2275.]
Walker, G.P.L., 2000, Basaltic volcanoes and volcanic systems, in Sigurdsson, H., ed., Encyclopedia of volcanoes: New York, Academic Press, p. 283-289.

Welhan, J.A., Farabaugh, R.L., Merrick, M.J., and Anderson, S.R., 2007, Geostatistical modeling of sediment abundance in a heterogeneous basalt aquifer at the Idaho National Laboratory, Idaho: U.S. Geological Survey Scientific Investigations Report 2006-5316 (DOE/ID-22201), 32 p.

Welhan, J.A., Johannesen, C.M., Davis, L.L., Reeves, K.S., and Glover, J.A., 2002, Overview and synthesis of lithologic controls on aquifer heterogeneity in the eastern Snake River Plain, Idaho, in Bonnichsen, Bill, White, C.M., and McCurry, M.O., eds., Tectonic and magmatic evolution of the Snake River Plain Volcanic Province: Idaho Geological Survey Bulletin 30, p. 435-460.

Wetmore, P.H., and Hughes, S.S., 1997, Change in magnitude of basaltic magmatism determined from model morphologies of subsurface quaternary lavas at the Idaho National Engineering and Environmental Laboratory, Idaho: Geological Society of America Abstracts with Programs, v. 29 , no. 6 , p. 365 .

Wetmore, P.H., Hughes, S.S., Rodgers, D.W., and Anderson, S.R., 1999, Late Quaternary constructional development of the Axial Volcanic Zone, eastern Snake River Plain, Idaho: Geological Society of America Abstracts with Programs, v. 31 , no. 4 , p. 61 . 



\section{Appendix A. Paleomagnetic Inclination Values and Basalt Flows from Coreholes in the Eastern Snake River Plain Aquifer at the Idaho National Laboratory}

Corehole paleomagnetic information for tables A1-A27 was originally published in Champion and others (2011) and Hodges and Champion (2016). Previously unpublished flow unit determinations for basalt that makes up the aquifer are presented in tables A1-A27; depth and paleomagnetic data for corehole USGS 144 are presented in table A28. Tables are organized alphabetically by corehole name. Appendix A tables are Microsoft ${ }^{\mathbb{R}}$. Excel files and are available for download at https://doi.org/10.3133/sir20175148.

Information in the tables includes sample depth for flow units that make up the eastern Snake River Plain aquifer, and identifies sample groups that were used to determine average inclination for flows and 95 percent uncertainty. Each sample has a characteristic remanent inclination in degrees and an alternating-field demagnetization level in milliTeslas or an alternative demagnetization approach. Positive inclination values indicate normal paleomagnetic polarity, and negative inclination values indicate reversed paleomagnetic polarity.

Some coreholes are not vertical. In cases where the corehole deviates from vertical to the north or south, deviation correction values were obtained from geophysical deviation logs and applied to the paleomagnetic inclination data obtained from deviated samples to account for this variation from the vertical. Paleomagnetic inclination data, which were corrected for borehole deviation are in parenthesis, and the corrected values presented in the column to the right of the measured values. Deviation corrections were applied to the average paleomagnetic inclination of sample groups.

Petrographic boundaries denote a significant change in mineralogy in a flow. Unrecovered core and sediment intervals also were recorded in the depth column. Samples that are labeled "NIIA" were not included in the average. The NIIA samples may have been thermally overprinted by overlying flows, tilted by endogenous inflation, struck by lightning when on the surface, or otherwise had their orientations disturbed so that they do not yield usable paleomagnetic inclination data.

Stepwise thermal demagnetizations were done only on samples of particular interest.

The paleomagnetic line fit (Li) indicates values derived from a line fit performed from a vector component diagram using a sequence, or all of the demagnetizations steps from each sample from that site. $\mathrm{A} \mathrm{Li}$ is accomplished through the simultaneous inspection of both horizontal and vertical 2D planes representing the diminishing of the remanent magnetic vector of a sample created through either progressive alternating field (AF) or thermal (Th) demagnetization. A straight line is regressed through those demagnetization steps which align toward a zero strength magnetization in both 2D planar views (Kirschvink, 1980).

Measurements are in feet and milliteslas because the raw data were collected using these units of measure. 

Publishing support provided by the U.S. Geological Survey Science Publishing Network, Tacoma Publishing Service Center

For more information concerning the research in this report, contact the Director, Idaho Water Science Center

U.S. Geological Survey

230 Collins Road

Boise, Idaho 83702

https://id.water.usgs.gov 
